\title{
Ligand Gaussian accelerated molecular dynamics (LiGaMD): Characterization of ligand binding thermodynamics and kinetics
}

\author{
Yinglong Miao*, Apurba Bhattarai, Jinan Wang \\ Center for Computational Biology and Department of Molecular Biosciences, University of \\ Kansas, Lawrence, KS 66047, USA \\ * Corresponding email: miao@ku.edu
}




\begin{abstract}
Calculations of ligand binding free energies and kinetic rates are important for drug design. However, such tasks have proven challenging in computational chemistry and biophysics. To address this challenge, we have developed a new computational method "LiGaMD", which selectively boosts the ligand non-bonded interaction potential energy based on the Gaussian accelerated molecular dynamics (GaMD) enhanced sampling technique. Another boost potential could be applied to the remaining potential energy of the entire system in a dual-boost algorithm (LiGaMD_Dual) to facilitate ligand binding. LiGaMD has been demonstrated on host-guest and protein-ligand binding model systems. Repetitive guest binding and unbinding in the $\beta$ cyclodextrin host were observed in hundreds-of-nanosecond LiGaMD simulations. The calculated binding free energies of guest molecules with sufficient sampling agreed excellently with experimental data $(<1.0 \mathrm{kcal} / \mathrm{mol}$ error). In comparison with previous microsecond-timescale conventional molecular dynamics simulations, accelerations of ligand kinetic rate constants in LiGaMD simulations were properly estimated using Kramers' rate theory. Furthermore, LiGaMD allowed us to capture repetitive dissociation and binding of the benzamidine inhibitor in trypsin within $1 \mu$ s simulations. The calculated ligand binding free energy and kinetic rate constants compared well with the experimental data. In summary, LiGaMD provides a promising approach for characterizing ligand binding thermodynamics and kinetics simultaneously, which is expected to facilitate computer-aided drug design.
\end{abstract}

Keywords: Ligand Gaussian accelerated molecular dynamics (LiGaMD), enhanced sampling, ligand binding, thermodynamics, free energy, kinetic rate constants. 


\section{Introduction}

Free energy calculations of protein-ligand binding have been central to computational chemistry and biophysics research for the last several decades ${ }^{1}$. This is largely motivated by designing potent drug molecules with high binding free energies in the pharmaceutical industry ${ }^{2}$. A number of computational methods that have been developed in the field include thermodynamic integration $(\mathrm{TI})^{3}$, free energy perturbation $(\mathrm{FEP})^{4}$, double decoupling method ${ }^{5}$, umbrella sampling ${ }^{6}$, steered molecular dynamics (SMD) ${ }^{7}$, funnel metadynamics ${ }^{8}$, molecular mechanics Poisson-Boltzmann surface area and generalized Born surface area (MM/PBSA and MM/GBSA) ${ }^{9}$, and so on. Ligand binding free energy calculations have also been the focus of Statistical Assessment of Modeling of Proteins and Ligands (SAMPL) ${ }^{10}$ and Drug Design Data Resource (D3R) ${ }^{11}$ community challenges.

Kinetics of ligand binding have recently been recognized to be potentially more relevant for drug design. In particular, the dissociation rate constant that determines the drug residence time appears to better correlate with drug efficacy than the binding free energy ${ }^{12}$. However, ligand kinetic rates have proven even more difficult to compute than the binding free energies, largely due to slow processes of ligand binding and dissociation over long timescales ${ }^{12 b}$. With remarkable advances in supercomputing and method developments, molecular dynamics (MD) simulations using specialized hardware ${ }^{13}$, Markov state model (MSM) $)^{14}$ and enhanced sampling ${ }^{15}$ have extended our capabilities to capture spontaneous ligand binding to target proteins. A number of enhanced sampling methods, including metadynamics ${ }^{16}$, random acceleration $\mathrm{MD}^{17}, \mathrm{SMD}^{18}$, weighted ensemble ${ }^{19}$ and selectively scaled $\mathrm{MD}^{20}$ have also been able to dissociate ligand molecules from the proteins. However, it remains extremely challenging to simulate repetitive 
ligand binding and dissociation processes, precluding accurate calculations of ligand kinetic rate constants.

With relatively small size and reduced complexity, host-guest binding systems has served as models of protein-ligand binding in SAMPL challenges ${ }^{10}$. The studied hosts include the cucurbit[7]uril (CB7), octa-acid, and CBClip and cyclodextrin (CD). These hosts are compounds that are typically much smaller than proteins but still large enough to bind guest molecules through non-covalent interactions, which share common characteristics as protein-ligand binding. Hostguest systems thus represent model systems for testing and improving free energy calculation methods. In addition to thermodynamics, Chang and co-workers have investigated kinetics of fast guest binding and dissociation in the $\beta-C D$ host $^{21}$. Tens to hundreds of repetitive guest binding and dissociation events were observed in microsecond-timescale MD simulations, which enabled comprehensive characterization of both thermodynamics and kinetics of the host-guest binding.

The $\mathrm{MSM}^{22}$ and metadynamics ${ }^{8,23}$ have been applied to investigate the thermodynamics and kinetics of protein-ligand binding, using particularly the benzamidine inhibitor binding to trypsin as a model system. Multiple metadynamics trajectories with a total of $5 \mu$ s simulation time were obtained to predict the ligand unbinding pathways and dissociation rate constant, $k_{\text {off. }}$ The calculated $k_{\text {off }}=9.1 \pm 2.5 \mathrm{~s}^{-1}$ was suggested to be in agreement of the experimental value $k_{\text {off }}^{\text {exp }}=$ $600 \pm 300 \mathrm{~s}^{-1}$. Separate funnel metadynamics simulations also allowed calculations of ligand binding free energies, in particular $-8.5 \pm 0.7 \mathrm{kcal} / \mathrm{mol}$ for the trypsin-benzamidine system ${ }^{8}$. The MSM built with $150 \mu$ s MD simulation data provided a complex picture of ligand binding kinetics and protein conformational plasticity ${ }^{22}$. The simulation predicted $k_{\mathrm{off}}=131 \pm 109 \times 10^{2} \mathrm{~s}^{-1}$ appeared to be faster than the experimental value, while the predicted binding rate constant $k_{\mathrm{on}}=6.4 \pm 1.6 \times$ $10^{7} \mathrm{M}^{-1} \cdot \mathrm{s}^{-1}$ compared well with the experimental value of $k_{o n}^{\text {exp }}=2.9 \times 10^{7} \mathrm{M}^{-1} \cdot \mathrm{s}^{-1}$. Repetitive ligand 
binding and unbinding in trypsin were also captured using a selective integrated-temperingsampling molecular dynamics (SITSMD) method recently ${ }^{24}$. The binding free energy of benzamidine in trypsin was estimated as $-5.06 \mathrm{kcal} / \mathrm{mol}$, but the ligand kinetic rates were not calculated from the SITSMD simulations.

Here, we present a new computational method called ligand Gaussian accelerated molecular dynamics ("LiGaMD"), which enables us to simulate repetitive ligand binding and unbinding, and thus characterize both thermodynamics and kinetics of ligand binding simultaneously. Gaussian accelerated molecular dynamics (GaMD) is an enhanced sampling computational technique that works by adding a harmonic boost potential to smooth the biomolecular potential energy surface. ${ }^{25} \mathrm{GaMD}$ greatly reduces energy barriers and accelerates biomolecular simulations by orders of magnitude. ${ }^{26}$ GaMD does not require pre-defined collective variables or reaction coordinates. Compared with the enhanced sampling methods that rely on careful selection of the collective variables, GaMD is of particular advantage for studying complex biological processes such as ligand binding to proteins ${ }^{27}$. Moreover, because the boost potential follows a Gaussian distribution, biomolecular free energy profiles can be properly recovered through cumulant expansion to the second order. ${ }^{25}$ GaMD builds on the previous accelerated MD (aMD) method $^{28}$, but solves its energetic reweighting problem ${ }^{29}$ for free energy calculations of large biomolecules. GaMD has been implemented in the widely used $\mathrm{AMBER}^{25,30} \mathrm{NAMD}^{31}$ and GENESIS $^{32}$ packages. GaMD has successfully revealed physical pathways and mechanisms of protein folding and ligand binding, which are consistent with experiments and long-timescale conventional MD (cMD) simulations. ${ }^{25,31,33}$ It has also been applied to characterize proteinprotein $^{34}$, protein-membrane, ${ }^{35}$ and protein-nucleic acid ${ }^{36}$ interactions. 
Building upon GaMD, we have developed LiGaMD for more efficient sampling simulations of protein-ligand binding and unbinding processes. In LiGaMD, the non-bonded electrostatic and van der Waals interactions between the bound ligand and protein/environment are selectively boosted to enable ligand dissociation. In this context, selective acceleration has been found useful in previous enhanced sampling techniques, including the selective aMD ${ }^{37}$, selectively scaled $\mathrm{MD}^{20}$, essential energy space random walk ${ }^{38}$, replica exchange solute tempering $\left(\right.$ REST) ${ }^{39}$ and REST2 ${ }^{40}$. In addition, a number of unbound ligand molecules are added in the solvent and another boost potential is applied on these ligand molecules, the protein and solvent in a dual-boost LiGaMD (LiGaMD_Dual) algorithm to facilitate ligand rebinding.

The LiGaMD method has been demonstrated on host-guest and protein-ligand binding model systems. Repetitive guest binding and unbinding in the $\beta$-CD host have been observed in hundreds-of-nanosecond LiGaMD simulations. The LiGaMD predicted guest binding free energies and kinetic rate constants agree well with those from previous cMD simulations ${ }^{21}$ and experimental data. Furthermore, LiGaMD has also allowed us to capture multiple dissociation and rebinding events of the benzamidine inhibitor in trypsin within $1 \mu$ s simulations. The reweighted ligand binding free energy and kinetic rate constants compared well with the experimental data.

\section{Methods}

\section{Ligand Gaussian Accelerated Molecular Dynamics (LiGaMD)}

Gaussian accelerated molecular dynamics (GaMD) is an enhanced sampling technique that works by adding a harmonic boost potential to smooth biomolecular potential energy surface and reduce the system energy barriers ${ }^{25}$. Details of the GaMD method have been described in previous 
studies $^{25,31,33 a}$. A brief summary is provided in Appendix A. Here, we develop a new ligand GaMD (LiGaMD) method for more efficient sampling of protein-ligand binding.

We consider a system of ligand $L$ binding to a protein $P$ in a biological environment $E$. The system comprises of $N$ atoms with their coordinates $r \equiv\left\{\vec{r}_{1}, \cdots, \vec{r}_{N}\right\}$ and momenta $p \equiv$ $\left\{\vec{p}_{1}, \cdots, \vec{p}_{N}\right\}$. The system Hamiltonian can be expressed as:

$$
H(r, p)=K(p)+V(r)
$$

where $K(p)$ and $V(r)$ are the system kinetic and total potential energies, respectively. Next, we decompose the potential energy into the following terms:

$$
\begin{aligned}
& V(r)=V_{P, b}\left(r_{P}\right)+V_{L, b}\left(r_{L}\right)+V_{E, b}\left(r_{E}\right) \\
& +V_{P P, n b}\left(r_{P}\right)+V_{L L, n b}\left(r_{L}\right)+V_{E E, n b}\left(r_{E}\right) \\
& +V_{P L, n b}\left(r_{P L}\right)+V_{P E, n b}\left(r_{P E}\right)+V_{L E, n b}\left(r_{L E}\right) .
\end{aligned}
$$

where $V_{P, b}, V_{L, b}$ and $V_{E, b}$ are the bonded potential energies in protein $P$, ligand $L$ and environment $E$, respectively. $V_{P P, n b}, V_{L L, n b}$ and $V_{E E, n b}$ are the self non-bonded potential energies in protein $P$, ligand $L$ and environment $E$, respectively. $V_{P L, n b}, V_{P E, n b}$ and $V_{L E, n b}$ are the non-bonded interaction energies between $P-L, P-E$ and $L-E$, respectively. According to classical molecular mechanics force fields ${ }^{41}$, the non-bonded potential energies are usually calculated as:

$$
V_{n b}=V_{e l e c}+V_{v d W}
$$

Where $V_{\text {elec }}$ and $V_{v d W}$ are the system electrostatic and van der Waals potential energies. Presumably, ligand binding mainly involves the non-bonded interaction energies of the ligand, $V_{L, n b}(r)=V_{L L, n b}\left(r_{L}\right)+V_{P L, n b}\left(r_{P L}\right)+V_{L E, n b}\left(r_{L E}\right)$. In LiGaMD, we add a boost potential selectively to the ligand non-bonded potential energy as: 


$$
\Delta V_{L, n b}(r)=\left\{\begin{aligned}
\frac{1}{2} k_{L, n b}\left(E_{L, n b}-V_{L, n b}(r)\right)^{2}, & V_{L, n b}(r)<E_{L, n b} \\
0, & V_{L, n b}(r) \geq E_{L, n b}
\end{aligned}\right.
$$

where $E_{L, n b}$ is the threshold energy for applying boost potential and $k_{L, n b}$ is the harmonic constant. For simplicity, the subscript of $\Delta V_{L, n b}(r), E_{L, n b}$ and $k_{L, n b}$ is dropped in the following. The LiGaMD simulation parameters are derived similarly as in the previous GaMD algorithm (Appendix A). When $E$ is set to the lower bound $E=V_{\max }, k_{0}$ can be calculated as:

$$
k_{0}=\min \left(1.0, k_{0}^{\prime}\right)=\min \left(1.0, \frac{\sigma_{0}}{\sigma_{V}} \frac{V_{\max }-V_{\min }}{V_{\max }-V_{a v g}}\right) .
$$

Alternatively, when the threshold energy $E$ is set to its upper bound $E=V_{\min }+\frac{1}{k}, k_{0}$ is set to:

$$
k_{0}=k_{0}^{\prime \prime} \equiv\left(1-\frac{\sigma_{0}}{\sigma_{V}}\right) \frac{V_{\max }-V_{\min }}{V_{a v g}-V_{\min }}
$$

if $k_{0}^{\prime \prime}$ is found to be between 0 and 1 . Otherwise, $k_{0}$ is calculated using Eqn. (5).

Next, one can add multiple ligand molecules in the solvent to facilitate ligand binding to proteins in MD simulations ${ }^{13 a, 42}$. This is based on the fact that the ligand binding rate constant $k_{\mathrm{on}}$ is inversely proportional to the ligand concentration. The higher the ligand concentration, the faster the ligand binds, provided that the ligand concentration is still within its solubility limit. In addition to selectively boosting the bound ligand to accelerate its dissociation, another boost potential could thus be applied on the unbound ligand molecules, protein and solvent to facilitate ligand rebinding. The second boost potential is calculated using the total system potential energy other than the nonbonded potential energy of the bound ligand as:

$$
\Delta V_{D}(r)=\left\{\begin{aligned}
\frac{1}{2} k_{D}\left(E_{D}-V_{D}(r)\right)^{2}, & V_{D}(r)<E_{D} \\
0, & V_{D}(r) \geq E_{D}
\end{aligned}\right.
$$


where $E_{D}$ and $k_{D}$ are the corresponding threshold energy for applying the second boost potential and the harmonic constant, respectively. This leads to dual-boost LiGaMD (LiGaMD_Dual) with the total boost potential $\Delta V(r)=\Delta V_{L, n b}(r)+\Delta V_{D}(r)$.

\section{Ligand Binding Free Energy Calculations from 3D Potential of Mean Force}

We calculate ligand binding free energy from 3D potential of mean force (PMF) of ligand displacements from the target protein as the following ${ }^{43}$ :

$$
\Delta G^{0}=-\Delta W_{3 D}-R T \operatorname{Ln} \frac{V_{b}}{V_{0}}
$$

where $V_{0}$ is the standard volume, $V_{b}=\int_{b} e^{-\beta W(r)} d r$ is the average sampled bound volume of the ligand with $\beta=1 / k_{B} T, k_{B}$ is the Boltzmann constant, $T$ is the temperature, and $\Delta W_{3 D}$ is the depth of the $3 \mathrm{D}$ PMF. $\Delta W_{3 D}$ can be calculated by integrating Boltzmann distribution of the $3 \mathrm{D}$ PMF $W(r)$ over all system coordinates except the $\mathrm{x}, \mathrm{y}, \mathrm{z}$ of the ligand:

$$
\Delta W_{3 D}=-R T \operatorname{Ln} \frac{\int_{u} e^{-\beta W(r)} d r}{\int_{u} d r}
$$

where $V_{u}=\int_{u} d r$ is the sampled unbound volume of the ligand. The exact definitions of the bound and unbound volumes $V_{b}$ and $V_{u}$ are not important as the exponential average cut off contributions far away from the PMF minima ${ }^{43 b}$. A python script PyReweighting-3D.py is freely available in the PyReweighting tool kit (http://miao.compbio.ku.edu/PyReweighting/) for calculating the 3D PMF and associated ligand binding free energies. It works for both cMD (without energetic reweighting) and enhanced sampling simulations using LiGaMD with energetic reweighting (Appendix B). 


\section{Ligand Binding Kinetics obtained from Reweighting of LiGaMD Simulations}

Provided sufficient sampling of repetitive ligand dissociation and binding in simulations, one can record the time periods and calculate their averages for the ligand found in the bound $\left(\tau_{B}\right)$ and unbound $\left(\tau_{U}\right)$ states from the simulation trajectories. The $\tau_{B}$ corresponds to residence time in drug $\operatorname{design}^{44}$. Then the ligand dissociation and binding rate constants $\left(k_{\text {off }}\right.$ and $\left.k_{\text {on }}\right)$ were calculated as:

$$
\begin{gathered}
k_{o f f}=\frac{1}{\tau_{B}} . \\
k_{o n}=\frac{1}{\tau_{B} \cdot[L]},
\end{gathered}
$$

where $[\mathrm{L}]$ is the ligand concentration in the simulation system.

Two algorithms were implemented using the transition state theory (TST) and Kramers' rate theory for reweighting kinetics of the LiGaMD simulations. According to Kramers' Rate Theory (Appendix C), the rate of a chemical reaction in the large viscosity limit is calculated as ${ }^{26}$ :

$$
k_{R} \cong \frac{2 \pi w_{m} w_{b}}{\xi} e^{-\Delta F / k_{B} T}
$$

where $w_{m}$ and $w_{b}$ are frequencies of the approximated harmonic oscillators (also referred to as curvatures of free energy surface ${ }^{45}$ ) near the energy minimum and barrier, respectively, $\xi$ is the apparent friction coefficient and $\Delta F$ is the free energy barrier of transition. The apparent friction coefficient $\xi$ is related to the diffusion coefficient $D$ with $\xi=k_{B} T / D$. The apparent diffusion coefficient $D$ can be obtained by dividing the kinetic rate calculated directly using the transition time series collected directly from simulations by that using the probability density solution of the Smoluchowski equation $^{46}$ (Appendix C). In order to reweight ligand kinetics from the LiGaMD simulations using the Kramer's rate theory, the free energy barriers of ligand binding and dissociation are calculated from the original (reweighted, $\Delta \boldsymbol{F}$ ) and modified (no reweighting, $\Delta \boldsymbol{F}^{*}$ ) 
PMF profiles, similarly for curvatures of the reweighed $(w)$ and modified $\left(w^{*}\right.$, no reweighting) PMF profiles near the guest bound ("B") and unbound ("U”) low-energy wells and the energy barrier ("Br"), and the ratio of apparent diffusion coefficients from LiGaMD simulations without reweighting (modified, $D^{*}$ ) and with reweighting $(D)$. The resulting numbers are then plugged into Eq. (11) to estimate accelerations of the ligand binding and dissociation rates during LiGaMD simulations (Appendix $\mathbf{C})^{26}$, which allows us to recover the original kinetic rate constants.

In comparison, the rate of a chemical reaction in the transition state theory (TST) is calculated as $^{47}$ :

$$
k_{T S T} \cong e^{-\Delta F / k_{B} T}
$$

Only the energy barriers of ligand binding and dissociation need to be calculated from the original (reweighted, $\Delta \boldsymbol{F}$ ) and modified (no reweighting, $\Delta \boldsymbol{F}^{*}$ ) free energy profiles for estimating the accelerations and recovering the ligand kinetic rate constants.

\section{Host-Guest Binding Simulations}

GaMD simulations were performed on the binding of two guest molecules (aspirin and 1-butanol) to the $\beta-C D$ host using the same input files as in a previous study by Tang and Chang ${ }^{21}$. The CD host were modeled with both the GAFF and q4MD force fields. The guest molecules were modeled with GAFF. The LiGaMD and dual-boost LiGaMD (LiGaMD_Dual) simulations were compared with GaMD simulations using the previous algorithms ${ }^{25,33 \mathrm{~b}}$, including the total potential boost GaMD (GaMD_Tot), dual-boost GaMD (GaMD_Dual) ${ }^{25}$, non-bonded potential boost GaMD (GaMD_NB) and non-bonded dual-boost GaMD (GaMD_Dual_NB) $)^{33 b}$ simulations, as well as the previous long microsecond-timescale cMD simulations ${ }^{21}$ (Tables 1 and S1). 
With the same restart files as used for running the cMD production simulations ${ }^{21}$, the GaMD simulations proceeded with $1 \mathrm{~ns}$ short cMD to collect potential statistics, $20 \mathrm{~ns}$ GaMD equilibration after adding the boost potential and then three independent $300 \mathrm{~ns}$ production runs. GaMD production frames were saved every 0.1 ps for analysis. The $\mathrm{VMD}^{48}$ and CPPTRAJ ${ }^{49}$ tools were used for simulation analysis. The number of host-guest dissociation and binding events $\left(N_{D}\right.$ and $\left.N_{B}\right)$ and the guest binding and unbinding time periods $\left(\tau_{B}\right.$ and $\left.\tau_{U}\right)$ were recorded from individual simulations (Table S2). With high fluctuations, $\tau_{B}$ and $\tau_{U}$ were recorded for only the time periods longer than $1 \mathrm{~ns}$ as applied in analysis of the previous cMD simulations ${ }^{21}$. For systems with more than one time of guest dissociation and binding in each of the individual simulations, 1D, 2D and 3D PMF profiles, as well as the host-guest binding free energies, were calculated through energetic reweighting of the GaMD simulations. The center-of-mass distances between the host and guests $\left(\mathrm{d}_{\mathrm{HG}}\right)$ and the host radius of gyration $\left(R_{g}\right)$ were chosen as reaction coordinates for calculating the 1D PMF profiles. 2D PMF profiles of $\left(\mathrm{d}_{\mathrm{HG}}, R_{g}\right)$ were also calculated to analyze conformational changes of the CD host upon guest binding. The bin size was set to $0.5 \AA$ for the $\mathrm{d}_{\mathrm{HG}}$ and $0.05 \AA$ for the $R_{g}$. The cutoff of the number of simulation frames in one bin for reweighting was set to 500 in 1D and 2D PMF calculations. The 3D PMF profiles of guest displacements from the $\mathrm{CD}$ host in the $\mathrm{X}, \mathrm{Y}$ and $\mathrm{Z}$ directions were further calculated from the LiGaMD simulations. The bin sizes were set to $1 \AA$ in the $\mathrm{X}, \mathrm{Y}$ and $\mathrm{Z}$ directions. The cutoff for the number of simulation frames in one bin for reweighting the 3D PMF was set to 10 for LiGaMD simulations and 50 for LiGaMD_Dual simulations. The host-guest binding free energies $(\Delta G)$ were calculated using the reweighted 3D PMF profiles and compared with those from previous cMD simulations and experimental data ${ }^{21}$ (Table S1). In addition, accelerations of the ligand dissociation and binding 
rate constants ( $k_{\text {on }}$ and $\left.k_{\text {off }}\right)$ in LiGaMD simulations were analyzed using the TST and Kramers' rate theory as described above (Table S3).

\section{Protein-Ligand Binding Simulations}

LiGaMD simulations using the dual-boost scheme were performed on benzamidine binding to the trypsin protein. The X-ray crystal structure of benzamidine-bound trypsin (PDB ID: $3 \mathrm{PTB}^{50}$ ) was used with the calcium ion and water molecules kept. The missing hydrogen atoms were added using by the tleap module in AMBER ${ }^{51}$. The general Amber force field ${ }^{52}$ and the AMBER ff14SB force field ${ }^{53}$ were used for the ligand and protein, respectively. Atomic partial charges of benzamidine were obtained through B3LYP/6-31G* quantum calculations of the electrostatic potential, for which the RESP charges ${ }^{54}$ were fitted using the antechamber program ${ }^{51}$. The system was neutralized by adding a number of counter ions $\left(\mathrm{Cl}^{-}\right)$and immersed in a cubic TIP3P water ${ }^{55}$ box, which was extended $13 \AA$ from the protein-ligand complex. Testing simulations were performed on protonation of the protein active-site residue His57 at the $\mathrm{N}_{\delta}$ atom or the $\mathrm{N}_{\varepsilon}$ atom. Results showed that benzamidine could bind to the protein target site as in the X-ray conformation with the $\mathrm{N}_{\delta}$ atom protonated, but not with atom $\mathrm{N}_{\varepsilon}$ protonated. Therefore, the protein residue His57 was protonated at the $\mathrm{N}_{\delta}$ atom. Moreover, a total of 10 ligand molecules (one in the X-ray bound conformation and another nine placed randomly in the solvent) were included in the system to facilitate ligand binding. This design was based on the fact that the ligand binding rate constant $k_{\mathrm{on}}$ is inversely proportional to the ligand concentration. The higher the ligand concentration, the faster the ligand binds, provided that the ligand concentration is still within its solubility limit.

The built simulation system was energy minimized with $1 \mathrm{kcal} / \mathrm{mol} / \AA^{2}$ constraints on the heavy atoms of the protein and ligand, including the steepest descent minimization of 5,000 steps 
followed by a conjugate gradient minimization of 5,000 steps. The system was then heated from 0 $\mathrm{K}$ to $300 \mathrm{~K}$ for $200 \mathrm{ps}$. It was further equilibrated using the NVT ensemble at $300 \mathrm{~K}$ for 800 ps and the NPT ensemble at $300 \mathrm{~K}$ and 1 bar for $1 \mathrm{~ns}$ with $1 \mathrm{kcal} / \mathrm{mol} / \AA^{2}$ constraints on the heavy atoms of the protein and ligand, followed by 2 ns short cMD without any constraint. The LiGaMD simulations proceeded with $14 \mathrm{~ns}$ short $\mathrm{cMD}$ to collect the potential statistics, $54.6 \mathrm{~ns}$ GaMD equilibration after adding the boost potential and then five independent $1000 \mathrm{~ns}$ production runs. Initial testing simulations showed that when the threshold energy for applying boost potential to the ligand non-bonded energy was set to the lower bound (i.e., $E=V_{\max }$ ), the bound ligand maintained the X-ray conformation and did not dissociate. In comparison, when the threshold energy was set to the upper bound (i.e., $E=V_{\min }+1 / k$ ), it enabled high enough boost potential to dissociate the ligand from the protein. Therefore, the threshold energy for applying the ligand boost potential was set to the upper bound in the LiGaMD_Dual simulations. For the second boost potential that was applied to the system total potential energy other than the ligand non-bonded potential energy, sufficient acceleration was obtained to sample ligand rebinding by setting the threshold energy to the lower bound. LiGaMD_Dual production simulation frames were saved every 0.2 ps for analysis.

The $\mathrm{VMD}^{48}$ and CPPTRAJ ${ }^{49}$ tools were used for simulation analysis. The number of ligand dissociation and binding events $\left(N_{D}\right.$ and $\left.N_{B}\right)$ and the ligand binding and unbinding time periods ( $\tau_{B}$ and $\tau_{U}$ ) were recorded from individual simulations (Table S4). With high fluctuations, $\tau_{B}$ and $\tau_{U}$ were recorded for only the time periods longer than $5 \mathrm{~ns}$. The 1D, 2D and 3D PMF profiles, as well as the ligand binding free energy, were calculated through energetic reweighting of the LiGaMD_Dual simulations. The distance between the $\mathrm{N}$ atom in benzamidine and $\mathrm{CG}$ atom of Asp189 in trypsin was chosen as the reaction coordinate for calculating the 1D PMF profiles. The 
bin size was set to 1.0 A. 2D PMF profiles of the benzamidine:N - Asp189:CG and Trp215:NE Asp 189:CG atom distances were also calculated to analyze conformational changes of the protein upon ligand binding. The bin size was set to $1.0 \AA$ for the atom distances. The cutoff for the number of simulation frames in one bin was set to 500 for reweighting $1 \mathrm{D}$ and 2D PMF profiles. The 3D PMF profiles of guest displacements from the CD host in the $\mathrm{X}, \mathrm{Y}$ and $\mathrm{Z}$ directions were further calculated from the LiGaMD simulations. The bin sizes were set to $1 \AA$ in the $\mathrm{X}, \mathrm{Y}$ and $\mathrm{Z}$ directions. The cutoff of simulation frames in one bin for 3D PMF reweighting (ranging from 1100-4000 for five individual LiGaMD_Dual simulations) was set to the minimum number below which the calculated 3D PMF minimum will be shifted. The ligand binding free energies $(\Delta G)$ were calculated using the reweighted 3D PMF profiles. Furthermore, structural clustering was performed on frames of the diffusing ligand molecules from each $1 \mu$ s LiGaMD_Dual simulation trajectory using the Density Based Spatial Clustering of Applications with Noise (DBSCAN) algorithm $^{56}$ in CPPTRAJ ${ }^{49}$. The frames were sieved at a stride of 500 for clustering. The remaining frames were assigned to the closest cluster afterwards. The distance cutoff for DBSCAN clustering was set to $0.5 \AA$. The resulting structural clusters were reweighted to obtain energetically significant binding pathways of the ligand. In addition, the ligand dissociation and binding rate constants $\left(k_{o n}\right.$ and $\left.k_{o f f}\right)$ were calculated from the LiGaMD_Dual simulations with their accelerations analyzed using the Kramers' rate theory as described above (Table S5).

\section{Results}

\section{Thermodynamics of Host-Guest Binding}

For the $\beta$-cyclodextrin (CD) host as modeled with both the GAFF and q4MD force fields, all-atom GaMD simulations were performed to investigate the dissociation and binding of two guest 
molecules aspirin (Fig. 1A) and 1-butanol (Fig. 1B) using a number of different potential boost algorithms (Table 1). The center-of-mass distances between the host and guest molecules were monitored as a function of time to record the number of dissociation and binding events $\left(N_{D}\right.$ and $\left.N_{B}\right)$ in each of the $300 \mathrm{~ns}$ GaMD simulations. The new LiGaMD method especially using the dualboost algorithm (LiGaMD_Dual) showed significantly improved sampling of ligand binding compared with the previous GaMD algorithms, including the total potential boost GaMD (GaMD_Tot), dual-boost GaMD (GaMD_Dual), non-bonded potential boost GaMD (GaMD_NB) and non-bonded dual-boost GaMD (GaMD_Dual_NB) (Table 1). Repetitive binding and unbinding of guest molecules were observed in 300 ns LiGaMD and LiGaMD_Dual simulations, except for the binding of 1-butanol to CD that was modeled with the GAFF force field (Fig. S1). In comparison, no guest dissociation and/or binding was observed in one or more of the $300 \mathrm{~ns}$ GaMD simulations using the other algorithms (Table 1).

Provided improved sampling in the LiGaMD and LiGaMD_Dual simulations, we computed potential of mean force (PMF) free energy profiles to characterize the host-guest binding quantitatively (Fig. 1). The host-guest distance $\left(\mathrm{d}_{\mathrm{HG}}\right)$ was chosen as a reaction coordinate. The PMF profiles calculated from three 300 ns LiGaMD or LiGaMD_Dual simulations were compared with those from microsecond-timescale cMD simulations of CD using the GAFF force field with aspirin (Fig. 1C), CD using the GAFF force field with 1-butanol (Fig. 1D), CD using the q4MD force field with aspirin (Fig. 1E), and CD using the q4MD force field with 1-butanol (Fig. 1F). For binding of 1-butanol to CD modeled with GAFF, while $300 \mathrm{~ns}$ LiGaMD and LiGaMD_Dual simulations sampled the system unbound $\left(\mathrm{d}_{\mathrm{HG}}>\sim 15 \AA\right)$ and intermediate $\left(\mathrm{d}_{\mathrm{HG}}=\sim 10 \AA\right)$ states, being closely similar to the $6500 \mathrm{~ns}$ cMD simulations, the bound state $\left(\mathrm{d}_{\mathrm{HG}}=\sim 0.5 \AA\right)$ was poorly sampled in the LiGaMD simulations (Fig. 1D). This correlated with the above finding that the 
guest dissociation and binding were seldomly observed in these simulations (Table 1 and Figs. S1C-S1D). For the other three systems, $300 \mathrm{~ns}$ LiGaMD and LiGaMD_Dual simulations sampled the same bound $\left(\mathrm{d}_{\mathrm{HG}}=\sim 0.5-2 \AA\right)$, intermediate $\left(\mathrm{d}_{\mathrm{HG}}=\sim 10 \AA\right)$ and unbound $\left(\mathrm{d}_{\mathrm{HG}}>\sim 15 \AA\right)$ lowenergy states as in the microsecond timescale cMD simulations (Figs. 1C, 1E and 1F). Their global energy minima were all identified in the bound state. However, differences were found in the magnitudes of PMF profiles near the intermediate, unbound and energy barrier regions. Overall, the LiGaMD_Dual simulations showed better agreements with the long-timescale cMD simulations than LiGaMD, especially for binding of 1-butanol to CD modeled with q4MD (Fig. 1F). The LiGaMD_Dual provided the most efficient sampling of host-guest binding and dissociation events (Table 1 and Fig. S1) and the closest free energy profiles as compared with the reference cMD simulations (Fig. 1).

Next, we analyzed conformational changes of the CD host modeled with GAFF (Fig. 2) and q4MD (Fig. 3) upon guest binding. PMF profiles were calculated for the radius of gyration $\left(R_{g}\right)$ of the CD host modeled with GAFF from the cMD simulations in the ligand-free (apo), aspirin and 1-butanol binding forms (Fig. 2A). Two low-energy states, including "Compact" $\left(R_{g}=\sim 5.7\right.$ $\AA$ ) and "Open" ( $\left.R_{g}=\sim 5.9 \AA\right)$, were identified for the CD host. While the apo and 1-butanol bound CD predominantly adopted the compact conformational state, binding of aspirin biased conformational ensemble of CD towards the open state. In this regard, aspirin (Fig. 1A) appeared to be larger in size than 1-butanol (Fig. 1B). Furthermore, we calculated 2D profiles of ( $\left.\mathrm{d}_{\mathrm{HG}}, R_{g}\right)$ and identified low-energy conformational states of the system. For aspirin binding, three lowenergy states were found from the 9500 ns cMD simulation, including the "Bound (B)", "Intermediate (I)" and "Unbound (U)" states, in which the CD host adopted primarily the Open, Compact and Compact conformations, respectively (Figs. 2B and 2C). The 2D PMF profile of 
aspirin binding calculated from three $300 \mathrm{~ns}$ LiGaMD_Dual simulations combined was highly similar to that from $9500 \mathrm{~ns} \mathrm{cMD}$ simulation, depicting the same three low-energy conformational states (Figs. 2C and 2D). In comparison, only the Bound and Unbound low-energy states were clearly identified for binding of 1-butanol in the 2D PMF profiles of both 6500 ns cMD (Fig. 2E) and three 300 ns LiGaMD_Dual simulations (Fig. 2F), while the intermediate state was hardly observed with apparently a very shallow energy well as shown in the 1D PMF profiles (Fig. 1D).

Furthermore, we evaluated the effects of different force field parameters for the CD host. With the q4MD force field, the CD host adopted predominantly the Open conformation with $R_{\mathrm{g}}=$ $\sim 6.1 \AA$ in PMF profiles of both the apo and guest-bound forms, although it still sampled the Compact conformation with a shallow and broad energy well in the apo form (Fig. 3A). This was in contrast to the above findings that the CD host using the GAFF force field preferred the Compact conformation in the apo form, while guest binding especially aspirin induced the host to open (Fig. 2). Therefore, the usage of different force fields generated distinct structural dynamics and free energy profiles of host-guest binding. Nevertheless, consistent results were obtained from LiGaMD_Dual and cMD simulations provided the same system setup and force field. In this context, LiGaMD only enhanced conformational sampling of the studied systems, yielding similar results as obtained from significantly longer cMD simulations.

In $6000 \mathrm{~ns}$ cMD simulation of aspirin binding to $\mathrm{CD}$ modeled with q4MD, three lowenergy conformational states were identified from 2D PMF profiles of the host-guest distance $\mathrm{d}_{\mathrm{HG}}$ and host $R_{g}$, including the "Bound (B)", "Intermediate (I)" and "Unbound (U)" states, in which the CD host all adopted primarily the Open conformation (Figs. 3B and 3C). A closely similar 2D PMF profile was obtained from three $300 \mathrm{~ns}$ LiGaMD_Dual simulations combined (Figs. 3D). Similar 2D profiles were obtained for 1-butanol binding from three $300 \mathrm{~ns}$ LiGaMD_Dual 
simulations combined and $5000 \mathrm{~ns}$ cMD simulation, during which CD was open in both the guest bound and unbound states (Figs. 3E and 3F).

In addition to the 1D and 2D PMF profiles, we computed 3D PMF profiles of guest binding to the $\mathrm{CD}$ host in the $\mathrm{X}, \mathrm{Y}$ and $\mathrm{Z}$ directions and then the guest binding free energies (see details in Methods). The host-guest binding free energies calculated from LiGaMD and LiGaMD_Dual simulations were compared with those obtained from previous cMD simulations and experimental data (Tables 2 and S1). Compared with experimental data, LiGaMD_Dual provided generally more accurate estimates of the guest binding free energies than LiGaMD (Table 2), being consistent with the finding that improved sampling with more dissociation and binding events was observed in the LiGaMD_Dual simulations (Table 1). For aspirin, the binding free energy errors were reduced from $-1.16 \pm 0.03 \mathrm{kcal} / \mathrm{mol}$ to $0.85 \pm 0.18 \mathrm{kcal} / \mathrm{mol}$ with the $\mathrm{CD}$ host modeled from GAFF and from $-1.00 \pm 0.53 \mathrm{kcal} / \mathrm{mol}$ to $0.40 \pm 0.15 \mathrm{kcal} / \mathrm{mol}$ with $\mathrm{CD}$ modeled using q4MD. For 1-butanol, the binding free energy error was calculated from only the simulations with CD modeled using q4MD, during each of which multiple dissociation and binding events were observed (Table 1). The binding free energy error of 1-butanol decreased from $-1.17 \pm 0.61 \mathrm{kcal} / \mathrm{mol}$ in LiGaMD simulations to $0.33 \pm 0.21 \mathrm{kcal} / \mathrm{mol}$ in LiGaMD_Dual simulations (Table 2). The guest binding free energy errors from the LiGaMD_Dual simulations were less than $1 \mathrm{kcal} / \mathrm{mol}$ and mostly comparable to those from previous microsecond-timescale $(5-9.5 \mu \mathrm{s}) \mathrm{cMD}$ simulations, despite variations in the guest binding free energies calculated using two different algorithms from the cMD simulations $\left(\Delta G_{\text {comp } 1}\right.$ and $\left.\Delta G_{\text {comp } 2}\right)$ as adapted from Ref. ${ }^{\mathbf{2 1}}$.

In summary, the PMF profiles and binding free energies of guest molecules in the CD host calculated from hundreds-of-nanosecond LiGaMD_Dual simulations agreed excellently with those from experimental data and previous microsecond-timescale cMD simulations ${ }^{\mathbf{2 1}}$. Errors in 
the guest binding free energies were smaller than $1 \mathrm{kcal} / \mathrm{mol}$ from the LiGaMD_Dual simulations as compared with the experimental data. Therefore, both efficient enhanced sampling and accurate free energy calculations of host-guest binding were achieved through the LiGaMD_Dual simulations.

\section{Kinetics of Host-Guest Binding}

In addition to the thermodynamic free energies, kinetic dissociation and binding rate constants were further derived from the relevant LiGaMD and LiGaMD_Dual simulations and compared with those from cMD simulations (Table 3). While the binding rate constants of guest molecules decreased to $\sim 30 \%-90 \%$ of those calculated from cMD simulations ${ }^{21}$, the dissociation rate constants increased by $\sim 1.7-15$ times in LiGaMD simulations and $\sim 11-18$ times in LiGaMD_Dual simulations (Table 3). In this context, long-timescale cMD simulations with repetitive host-guest binding and unbinding were available for comparison. However, in most protein-ligand binding studies (e.g., the trypsin-benzamidine binding described below) such cMD simulations are often not available, due to the extreme challenge of sampling free ligand binding and dissociation over long timescales. In this regard, we sought to reweight kinetics of LiGaMD and recover the original kinetic dissociation and binding rate constants from only the LiGaMD enhanced sampling simulations as follows.

Two algorithms were implemented using the transition state theory (TST) and Kramers' rate theory for reweighting kinetics of the LiGaMD simulations (see details in Methods). In both algorithms, the energy barriers of ligand binding and dissociation were calculated from the original (reweighted) and modified (no reweighting) free energy profiles of the LiGaMD simulations. Since 
the reweighted PMF profiles were already obtained for the host-guest distances from the LiGaMD and LiGaMD_Dual simulations (Fig. 1), we also calculated the corresponding modified PMF profiles without energetic reweighting for comparative analysis (Fig. 4). Note that the bound state of 1-butanol in CD modeled with GAFF was poorly sampled in the LiGaMD and LiGaMD_Dual simulations (Figs. 1D, S1 and S2). This system was thus excluded for both binding free energy and kinetics calculations. For the other three systems, the energy barriers were significantly reduced in the modified PMF profiles of both the LiGaMD and LiGaMD_Dual simulations for guest dissociation (Fig. 4 and Table S3). In LiGaMD_Dual simulations of aspirin binding to CD modeled with GAFF, the dissociation free energy barrier $\left(\Delta F_{\text {off }}\right)$ decreased by $73 \%$ from $2.06 \pm$ $0.28 \mathrm{kcal} / \mathrm{mol}$ in the reweighted PMF profile to $0.59 \pm 0.37 \mathrm{kcal} / \mathrm{mol}$ in the modified PMF profile (Fig. 4B). In the other LiGaMD and LiGaMD_Dual simulations, $\Delta F_{\text {off }}$ mostly decreased by $\sim 50 \%$ (Fig. 4 and Table S3). Correspondingly, $k_{\text {off }}$ estimated using the TST increased by $\sim 9-22$ times in the LiGaMD simulations of aspirin and 1-butanol binding to $\mathrm{CD}$ and $\sim 6-41$ times in the LiGaMD_Dual simulations (Table 3). On the other hand, the free energy barrier for ligand binding $\left(\Delta F_{o n}\right)$ actually increased in all modified PMF profiles of the LiGaMD and LiGaMD_Dual simulations than in the reweighted profiles (Fig. 4 and Table S3). According the TST, the corresponding binding rate constant $\left(k_{o n}\right)$ decreased by a factor $\sim 0.1-0.4$ in LiGaMD simulations and $\sim 0.1-0.2$ in LiGaMD_Dual simulations (Table 3). Despite the slower binding, significant accelerations were achieved in the rate-limiting dissociation of guest molecules during LiGaMD and LiGaMD_Dual simulations. Therefore, the guest dissociation and binding appeared to be more balanced in LiGaMD than in cMD, thereby resulting in overall improved sampling.

For applying the Kramers' rate theory, curvatures of the reweighed $(w)$ and modified $\left(w^{*}\right.$, no reweighting) free energy profiles were further calculated near the guest bound ("B") and 
unbound ("U”) energy minima and the energy barrier ("Br"), as well as the ratio of apparent diffusion coefficients calculated from the LiGaMD and LiGaMD_Dual simulations with reweighting $(D)$ and without reweighting (modified, $D^{*}$ ) (Table S3). The resulting numbers were plugged into the Kramers' rate equation to estimate accelerations of the guest binding and dissociation rate constants. Particularly, the $k_{\text {off }}$ increased by $\sim 8-12$ times in LiGaMD simulations and 6-23 times in LiGaMD_Dual simulations (Table 3). The $k_{\text {on }}$ decreased by a factor of 0.020.55 in LiGaMD simulation. In LiGaMD_Dual simulations, $k_{\text {on }}$ remained the same for 1-butanol binding to $\mathrm{CD}$ modeled with $\mathrm{q} 4 \mathrm{MD}$, but also decreased by factors of 0.06 and 0.23 for aspirin binding to CD modeled with GAFF and q4MD force fields, respectively (Table 3).

In summary, accelerations of host-guest dissociation and binding obtained from LiGaMD and LiGaMD_Dual simulations were derived using the TST and Kramers' Rate Theory. Compared with cMD simulations, while the binding rates decreased to a certain extent, guest unbinding was significantly accelerated by $~ 1.7-15$ times in LiGaMD and 11-18 times in LiGaMD_Dual. Overall, the Kramers' rate theory provided more accurate estimates of the ligand kinetic rate accelerations in the LiGaMD simulations than the TST (Table 3). It was thus applied for reweighting of ligand kinetics in analysis of further LiGaMD simulations.

\section{Thermodynamics of Ligand Binding to the Trypsin Model Protein}

In addition to host-guest binding, LiGaMD was further tested on protein-ligand binding using trypsin as a model system. Initial testing simulations suggested the following setup for proper simulations of benzamidine binding to trypsin: First, residue His57 at the protein active site was protonated at the $\mathrm{N}_{\delta}$ atom rather than the default $\mathrm{N}_{\varepsilon}$ atom, which was also depicted for the catalytic 
triad at the active site of proteases ${ }^{57}$. Testing simulations with the $\mathrm{N}_{\varepsilon}$ atom protonated in residue His57 showed that the benzamidine inhibitor could not bind to the protein target site as in the Xray crystal conformation with $\sim 4.3 \AA$ minimum root-mean square deviation (RMSD). In comparison, simulations with $\mathrm{N}_{\delta}$ atom could capture repetitive binding of benzamidine to the target site with $\sim 1.0$ A minimum RMSD compared with the X-ray conformation (Fig. S3). Second, the threshold energy for applying boost potential to the ligand non-bonded interaction energy was set to the upper bound (i.e., $E=V_{\min }+1 / k$ ). This enabled high enough boost potential to dissociate the ligand from the protein active site. In comparison, the bound ligand maintained the X-ray conformation during $\sim 200 \mathrm{~ns}$ testing simulations with the threshold energy set to the lower bound (i.e., $E=V_{\max }$ ). Moreover, higher acceleration was observed for the bound ligand as the input parameter $\sigma_{0 P}$ was increased from $1.0 \mathrm{kcal} / \mathrm{mol}$ to $6.0 \mathrm{kcal} / \mathrm{mol}$ and the ligand started to dissociate from the target site during the LiGaMD equilibration simulation with $\sigma_{0 P}=4.0 \mathrm{kcal} / \mathrm{mol}(\mathbf{F i g}$. S3), which was thus used for production simulations. Third, a total of 10 ligand molecules (one in the X-ray bound conformation and another nine placed randomly in the solvent) were included in the system to facilitate ligand rebinding. This design was based on the fact that the ligand binding rate constant $k_{\mathrm{on}}$ is inversely proportional to the ligand concentration. The higher the ligand concentration, the faster the ligand binds, provided that the ligand concentration is still within its solubility limit. By applying the second boost potential to the unbound ligand molecules, protein and solvent, ligand rebinding could be sampled even during the LiGaMD equilibration simulations (Figs. S3D and S3F).

With the above settings, LiGaMD_Dual simulations were able to capture repetitive dissociation and binding of the benzamidine inhibitor in trypsin within $1 \mu$ s simulation time (Figs. 5A, S4 and S5 and Movies S1-S5). In five independent LiGaMD_Dual simulations as summarized 
in Table 4, the average of the GaMD boost potential $\Delta V$ was $\sim 21-22 \mathrm{kcal} / \mathrm{mol}$ with $\sim 4.2-4.3$ $\mathrm{kcal} / \mathrm{mol}$ standard deviation. The ligand dissociated for 3-11 times and rebound for 3-10 times during the five $1 \mu \mathrm{s}$ LiGaMD_Dual simulations. In the X-ray bound conformation, the distance between the $\mathrm{N}$ atom in benzamidine and $\mathrm{CG}$ atom of Asp189 in trypsin was $3.9 \AA$. During the LiGaMD_Dual simulations, the bound ligand dissociated from the protein and diffused into the bulk solvent with the benzamidine:N - ASP189:CG distance increased up to $\sim 60 \AA$ (Figs. 5A and S5), similarly for the ligand RMSD relative to the X-ray conformation (Fig. S4). Then after sufficient sampling of the bulk solvent space, one of the ten ligand molecules rebound to the protein with a salt bridge formed between the charged benzamidine and side chain of protein residue Asp189, for which the benzamidine:N - Asp189:CG distance and ligand RMSD dropped back to $\sim 3.9 \AA$ (Fig. 5A) and $\sim 1.0 \AA$ (Fig. S4), respectively. When the benzamidine:N Asp189:CG distance dropped below a threshold value (3.7 $\AA$ defined here), atomic coordinates, velocities and forces of the bound ligand were swapped with those of the original bound ligand in the simulation starting structure (denoted "Lig0"). Upon dissociation of Lig0 from the protein, the high concentration of ten ligand molecules in the solvent facilitated ligand rebinding to the protein. Through such cycles, repetitive dissociation and binding of the benzamidine ligand in trypsin were efficiently sampled in the LiGaMD_Dual simulations.

Next, we calculated PMF free energy profiles to characterize ligand binding to trypsin. Since plots of the benzamidine:N - Asp189:CG distance and ligand RMSD depicted the ligand binding processes similarly (Figs. S4 and S5) and extra efforts were needed to calculate symmetrycorrected RMSD of benzamidine, the PMF profiles were calculated for the benzamidine:N Asp189:CGdistance. The resulting reweighted and modified 1D PMF profiles were shown in Fig. 5B. In the reweighted PMF profile, three low-energy wells were identified for the ligand in the 
Bound, Intermediate and Unbound states, for which the benzamidine: $\mathrm{N}$ - Asp189:CG distance was centered around $\sim 4.5 \AA, \sim 15 \AA$ and $\sim 35 \AA$, respectively. A high energy barrier of $12.17 \pm 1.54$ $\mathrm{kcal} / \mathrm{mol}$ was observed for the ligand dissociation (Table S5). In the modified PMF profile without reweighting, the energy barrier decreased to $1.37 \pm 0.56 \mathrm{kcal} / \mathrm{mol}$ for ligand dissociation and the energy wells became significantly shallower for the ligand in the Bound, Intermediate and Unbound states. This justified enhanced sampling of protein-ligand binding and unbinding in the LiGaMD_Dual simulations.

Furthermore, we computed 2D PMF profiles to analyze conformational changes of the protein upon ligand binding. Upon close examination of the system trajectories, residue Trp215 and its associated loop in trypsin underwent the largest conformational changes during ligand binding (Movies S1-S5). In this regard, a distance between the $\mathrm{N}_{\varepsilon}$ atom in the Trp215 side chain and CG atom of Asp189 was selected as another reaction coordinate. The calculated reweighted and modified 2D PMF profiles of the benzamidine:N - Asp189:CG and Trp215:NE - Asp189:CG atom distances are plotted in Fig. 5C and 5D, respectively. Five 1000 ns LiGaMD_Dual simulations were combined for calculating the PMF profiles. The reweighted and modified 2D PMF profiles calculated from five individual LiGaMD_Dual simulations separately are also shown in Figs. S6 and S7, respectively.

In the reweighted 2D PMF, low-energy wells were identified for the trypsin-benzamidine system in the Bound, Intermediate I1, Intermediate I2, Unbound U1 and Unbound U2 states, for which the (benzamidine:N - Asp189:CG, Trp215:NE - Asp189:CG) atom distances were centered around $(4.0 \AA, 12.5 \AA),(17.0 \AA, 12.5 \AA),(7.5 \AA, 7.5 \AA),(35.0 \AA, 12.5 \AA)$ and $(35.0 \AA, 8.0 \AA)$, respectively (Fig. 5C). In the modified 2D PMF profile (Fig. 5D), low-energy wells were also identified for the system in the Bound, Intermediate I1, Unbound U1 and Unbound U2 states in 
similar locations as in the reweighted 2D PMF. A distinct low-energy well was identified for the system in an Intermediate I3 state in the modified PMF with (14.0 $\AA, 9.0 \AA)$ for the (benzamidine:N - Asp189:CG, Trp215:NE - Asp189:CG) atom distances, rather than the I2 state in the reweighted 2D PMF. These low-energy wells were also observed in the reweighed and modified PMF profiles of five individual LiGaMD_Dual simulations (Figs. S6 and S7).

Next, we extracted six representative low-energy conformations of the trypsinbenzamidine system as identified from the PMF profiles, including the "Bound" (Fig. 6A), “Intermediate I1" (Fig. 6B), “Intermediate I2” (Fig. 6C), “Intermediate I3” (Fig. 6D), “Unbound U1" (Fig. 6E), and "Unbound U2" (Fig. 6F). In the Bound conformational state, the benzamidine ligand bound to protein residue Asp189 in the S1 pocket as in the X-ray crystal structure. Protein residue Trp215 closed the S1* pocket, but left the $\mathrm{S} 1$ pocket open, which was located between the Asp189 loop and the Trp215 loop (Fig. 6A). In the Intermediate I1 state, the benzamidine ligand bound to a site out of the Trp215 "gate", being close to the His57-Asp102-Ser214 catalytic triad (Fig. 5B). In the Intermediate I3 state, residue Trp215 flipped its side chain to close the S1 pocket, while the $\mathrm{S}^{*}$ pocket became open with rearrangement of the Trp215 loop compared with the Xray structure. The benzamidine ligand bound to the open $\mathrm{S} 1$ * pocket (Fig. 6C). In the Intermediate I3 state, the side chain of protein residue Trp215 rotated to a conformation perpendicular to the Xray conformation such that both $\mathrm{S} 1$ and $\mathrm{S} 1 *$ pockets became open and the benzamidine ligand bound to a site in close proximity of Trp215 and catalytic residues Asp102 and Ser214 (Fig. 6D). In the Unbound U1 state, trypsin adopted a conformation similar to the X-ray structure with the $\mathrm{S} 1$ pocket open and the $\mathrm{S} 1$ * pocket closed by Trp215, while the benzamidine ligand was found far away from the protein (Fig. 6E). In the Unbound U2 state, trypsin flipped its side chain to close 
the S1 pocket, similar to the Intermediate I2 conformation, while the benzamidine ligand stayed far away from the protein (Fig. 6F).

In addition to the $1 \mathrm{D}$ and 2D PMF profiles, 3D PMF was calculated from each individual $1 \mu \mathrm{s}$ LiGaMD_Dual simulation of benzamidine binding to trypsin in terms of displacements of the benzamidine $\mathrm{N}$ atom from the $\mathrm{CG}$ atom in protein residue Asp189 in the $\mathrm{X}, \mathrm{Y}$ and $\mathrm{Z}$ directions. We then calculated the ligand binding free energy from each 3D reweighted PMF (see Methods). The average of resulting free energy values was $-6.13 \mathrm{kcal} / \mathrm{mol}$ and the standard deviation was $0.35 \mathrm{kcal} / \mathrm{mol}$. This was in excellent agreement with the experimental value of $-6.2 \mathrm{kcal} / \mathrm{mol}$ for the ligand binding free energy of benzamidine in $\operatorname{trypsin}^{58}$ (Table 5).

\section{Pathways and kinetics of Ligand Binding to Trypsin}

With accurate prediction of the ligand binding free energy, we analyzed the LiGaMD_Dual simulations further to determine the pathways and kinetic rate constants of benzamidine binding to trypsin. For each $1 \mu \mathrm{s}$ simulation trajectory, structural clustering was performed on snapshots of the diffusing ligand molecules using the DBSCAN algorithm and the resulting structural clusters were reweighted to obtain energetically significant pathways of the ligand (see Methods). Fig. 7 depicts the dissociation and binding pathways of the benzamidine ligand in trypsin obtained from the five individual LiGaMD_Dual simulations. The ligand clusters along each pathway were colored according to the reweighted PMF values in a blue $(0 \mathrm{kcal} / \mathrm{mol})$-white $(7.5 \mathrm{kcal} / \mathrm{mol})$-red $(15.0 \mathrm{kcal} / \mathrm{mol})$ scale. The lowest-energy ligand cluster was consistently identified at the target site with benzamidine forming ionic interaction with trypsin residue Asp189 as determined in the Xray crystal structure (Fig. 7). 
During the LiGaMD_Dual simulations, benzamidine first dissociated predominantly through the protein opening between the Asp189 loop and the Trp215 loop, denoted pathway "P1" (Fig. 7). Then benzamidine molecules could rebind spontaneously to trypsin. Negatively charged residues on the protein surface, including Glu70, Asp71, Glu77, Glu80 and Asp153, appeared to steer the positively charged ligand towards the enzyme catalytic site formed by residues His57Asp102-Ser214. This was consistent with previous findings of "electrostatic steering" in ligand recognition by proteins ${ }^{59}$. Subsequently, the ligand bound to the target site via two pathways, one being the pathway "P1" and the other connecting the $\mathrm{S} 1 *$ pocket and the Trp215 side chain gate as closed in the X-ray structure (denoted pathway "P1*") (Fig. 7). In Sim1 and Sim2, because the Trp215 side chain maintained the X-ray conformation and closed the $\mathrm{S} 1$ * pocket, the benzamidine ligand dissociated and bound to the S1 pocket repetitively through the P1 pathway (Figs. 7A and 7B and Movies S1 and S2). In the other three simulations Sim3-Sim5, the Trp215 side chain underwent conformational changes to open either the $\mathrm{S} 1$ or $\mathrm{S} 1 *$ pocket or both pockets. Benzamidine dissociated and bound to the protein via either of the $\mathrm{P} 1$ and $\mathrm{P} 1$ * pathways (Figs. 7C-7E and Movies S3-S5). Notably, binding of the ligand was able to switch from pathway P1* to pathway $\mathrm{P} 1$ via the $\mathrm{S} 1 *$ binding pocket to the final target site in the $\mathrm{S} 1$ pocket as observed near $286 \mathrm{~ns}$ in the Sim5 trajectory (Movie S5). In addition, two ligand molecules were found to bind both the $\mathrm{S} 1$ and $\mathrm{S} 1 *$ pockets simultaneously during $310.5 \mathrm{~ns}-333.5 \mathrm{~ns}$ in the Sim5 trajectory (Movie S5 and Fig. S5E).

To calculate kinetic rate constants of benzamidine binding to trypsin, we recorded the time periods for the ligand found in the bound $\left(\tau_{\mathrm{B}}\right)$ and unbound $\left(\tau_{\mathrm{U}}\right)$ states throughout the LiGaMD_Dual simulations (Table S4). Since a total of 10 ligand molecules and 10,478 water molecules were included in the simulation system, the ligand concentration was $0.053 \mathrm{M}$ in the 
simulations. Without reweighting of the LiGaMD_Dual simulations, the benzamidine ligand binding $\left(k_{o n}^{*}\right)$ and dissociation $\left(k_{o f f}{ }^{*}\right)$ rate constants were calculated as $1.58 \pm 1.09 \times 10^{8} \mathrm{M} \cdot \mathrm{s}^{-1}$ and $3.40 \pm 1.36 \times 10^{7} \mathrm{~s}^{-1}$, respectively.

Following the same protocol as described for analyzing the host-guest binding simulations, we reweighted the trypsin-benzamidine binding simulations to calculate acceleration factors of the ligand binding and dissociation (Table S5) and recover the original kinetic rate constants using the Kramers' rate theory. The original (reweighted) and modified PMF profiles of protein-ligand distance are shown in Fig. 5B. The dissociation free energy barrier $\left(\Delta F_{\text {off }}\right)$ decreased by $\sim 90 \%$ from $12.17 \pm 1.54 \mathrm{kcal} / \mathrm{mol}$ in the reweighted PMF profile to $1.37 \pm 0.56 \mathrm{kcal} / \mathrm{mol}$ in the modified PMF profile (Table S5). On the other hand, the free energy barrier for ligand binding $\left(\Delta F_{o n}\right)$ decreased from $3.04 \pm 2.04 \mathrm{kcal} / \mathrm{mol}$ in the reweighted profile to $2.40 \pm 0.41 \mathrm{kcal} / \mathrm{mol}$ in the modified PMF profile (Fig. 5B and Table S5). Furthermore, curvatures of the reweighed (w) and modified ( $w^{*}$, no reweighting) free energy profiles were calculated near the guest bound ("B”) and unbound ("U") low-energy wells and the energy barrier ("Br"), as well as the ratio of apparent diffusion coefficients calculated from the LiGaMD_Dual simulations with reweighting $(D)$ and without reweighting (modified, $D^{*}$ ) (Table S5). According to the Kramers' rate theory, the ligand binding and acceleration were accelerated by 13.76 and $9.62 \times 10^{7}$ times, respectively. Therefore, the reweighted $k_{\text {on }}$ and $k_{\text {off }}$ were calculated as $1.15 \pm 0.79 \times 10^{7} \mathrm{M}^{-1} \cdot \mathrm{s}^{-1}$ and $3.53 \pm 1.41 \mathrm{~s}^{-1}$, respectively. They were comparable to the experimental data ${ }^{58}$ of $k_{o n}^{\text {exp }}=2.9 \times 10^{7} \mathrm{M}^{-1} \cdot \mathrm{s}^{-1}$ and $k_{\text {off }}^{\text {exp }}$ $=600 \pm 300 \mathrm{~s}^{-1}$ (Table 5). 


\section{Discussion}

A new LiGaMD method has been developed to selectively boost the ligand non-bonded interaction potential energy, which enables enhanced sampling simulations of repetitive ligand dissociation and binding as demonstrated on the host-guest and protein-ligand binding model systems. LiGaMD provides a promising approach for simultaneously calculating the free energy and kinetic rate constants of ligand binding.

For host-guest binding, LiGaMD and dual-boost LiGaMD (LiGaMD_Dual) significantly improved the sampling efficiency compared with the previous GaMD_Tot, GaMD_Dual, GaMD_NB and GaMD_NB_Dual algorithms (Table 1). For simulations that captured multiple events of ligand binding and dissociation, the accuracy of calculated ligand binding free energies and kinetic rate constants was similar to those obtained from much longer cMD simulations as compared with the experimental data. Notably, the CD host exhibited distinct structural dynamics when modeled with the different GAFF and q4MD force fields. Nevertheless, similar results were obtained from LiGaMD and cMD simulations provided the same force field. Therefore, LiGaMD only enhanced conformational sampling of the studied systems, but did not change statistics related to the force field. Apart from enhanced sampling, it remains critical to develop accurate force fields for systems of our interest such as the proteins and ligand molecules.

For the trypsin model protein-ligand binding system, the threshold energy for applying LiGaMD boost potential to non-bonded potential energy of the bound ligand was set to the upper bound so that high enough acceleration was obtained to allow for ligand dissociation. Multiple ligand molecules (e.g., 10 in the present simulations) were included in the system to facilitate binding. This was based on the fact that higher ligand concentration would lead to faster binding 
rate constant $k_{\text {on. }}$ Multiple events of ligand dissociation and binding were observed in each of the five independent $1 \mu$ s LiGaMD_Dual simulations of the trypsin-benzamidine system. Trypsin residue Trp215 appeared to be a gate for ligand binding through the $\mathrm{P} 1$ and $\mathrm{P} 1 *$ pathways.

The low-energy conformational states (Fig. 6) and ligand pathways (Fig. 7) of the trypsinbenzamidine system identified from LiGaMD_Dual simulations were mostly consistent with those obtained from previous simulation studies, especially the $\mathrm{MSM}^{22}$. However, LiGaMD_Dual simulations further revealed two novel findings. First, two benzamidine molecules were able to bind both the $\mathrm{S} 1$ and $\mathrm{S} 1$ * pockets simultaneously during one of the five LiGaMD_Dual simulation trajectories (Movie S5). While this finding could result from a relatively high ligand concentration $(0.053 \mathrm{M})$ with 10 benzamidine molecules in the simulation system, such rare event is worthy further investigation in the future. Second, binding of the benzamidine ligand was able to switch from the P1* pathway to the P1 pathway in the LiGaMD_Dual simulations. The ligand bound to the S1 pocket via an intermediate site in the S1* pocket. LiGaMD_Dual appeared to provide improved sampling compared with MD simulations used in the MSM so that such binding process could be captured. This LiGaMD_Dual simulation finding also suggested that the final target binding site of benzamidine is located in the S1 pocket as determined in the X-ray crystal structure. Ligand binding in the X-ray conformational state corresponded the global free energy minimum in the calculated PMF profiles.

Furthermore, the ligand binding free energy calculated from five $1 \mu$ s LiGaMD_Dual simulations $\Delta G^{0}=-6.13 \pm 0.35 \mathrm{kcal} / \mathrm{mol}$ was in excellent agreement with the experimental value $\Delta G_{\text {exp }}^{0}=-6.2 \mathrm{kcal} / \mathrm{mol}$. The ligand kinetic rate constants calculated by reweighting of the LiGaMD_Dual simulations were $k_{\text {on }}=1.15 \pm 0.79 \times 10^{7} \mathrm{M}^{-1} \cdot \mathrm{s}^{-1}$ and $k_{\text {off }}=3.53 \pm 1.41 \mathrm{~s}^{-1}$. The 
binding rate constant $k_{\text {on }}$ agreed excellently with the experimental value $k_{\text {on }}^{\text {exp }}=2.9 \times 10^{7} \mathrm{M}^{-1} \cdot \mathrm{s}^{-1}$, while the dissociation rate constant $k_{\text {off }}$ appeared to be slower than the experimental value $k_{\text {off }}^{\text {exp }}=$ $600 \pm 300 \mathrm{~s}^{-1} 58$ (Table 5).

In comparison, the ligand dissociation rate constant estimated from five $1 \mu \mathrm{s}$ LiGaMD_Dual simulations $k_{\text {off }}=3.53 \pm 1.41 \mathrm{~s}^{-1}$ was similar to the value of $k_{\text {off }}=9.1 \pm 2.5 \mathrm{~s}^{-1}$ obtained from previous metadynamics simulations also performed at a total of $5 \mu$ sength $^{16 a}$. However, the benzamidine binding free energy calculated from LiGaMD_Dual simulations $\Delta G^{0}$ $=-6.13 \pm 0.35 \mathrm{kcal} / \mathrm{mol}$ was more accurate than the values of $8.5 \pm 0.7 \mathrm{kcal} / \mathrm{mol}$ obtained from separate funnel metadynamics simulations ${ }^{8}$ and $-5.06 \mathrm{kcal} / \mathrm{mol}$ from the SITSMD simulations ${ }^{24}$, as compared with experimental data $\Delta G_{\text {exp }}^{0}=-6.2 \mathrm{kcal} / \mathrm{mol}$. In this regard, previous MSM predicted accurate ligand binding free energy $\Delta G^{0}=-6.05 \pm 1 \mathrm{kcal} / \mathrm{mol}$ and binding rate constant $k_{\text {on }}=6.4 \pm 1.6 \times 10^{7} \mathrm{M}^{-1} \cdot \mathrm{s}^{-1}$, while the model predicted $k_{\text {off }}=131 \pm 109 \times 10^{2} \mathrm{~s}^{-1}$ appeared to be faster than the experimental value ${ }^{22}$. Both MSM and LiGaMD_Dual could be applied to calculate the ligand binding free energy and kinetic rate constants simultaneously, but more expensive and significantly longer simulations were needed for the $\operatorname{MSM}(\sim 150 \mu \mathrm{s}$ in total $)$ than for the LiGaMD_Dual simulations $(1 \mu \mathrm{s} \times 5)$.

In summary, LiGaMD provides a promising approach to calculate both free energy and kinetic rate constants of ligand binding simultaneously. The ligand binding free energy is calculated based on unconstrained enhanced sampling and 3D PMF profiles, being distinct from previous methods such as the $\mathrm{TI}^{3}, \mathrm{FEP}^{4}$ and funnel metadynamics ${ }^{8}$. Beyond thermodynamics, LiGaMD also presents a new approach to estimate the ligand kinetic dissociation and binding rate constants. While LiGaMD has been demonstrated on host-guest and trypsin protein-ligand binding model systems, the method awaits further testing on many other ligand binding systems, notably 
membrane proteins. Although a total number of 10 ligand molecules has been included in the trypsin-benzamidine simulations to facilitate ligand binding, it will be more systematic to optimize the number of ligand molecules for future simulations according to the ligand solubility. Furthermore, a distance cutoff has been implemented in the current LiGaMD to determine when a ligand molecule binds to the protein target site and higher boost potential will be applied accordingly to enable ligand dissociation. More metrics that can serve in this purpose for a wide range of ligand binding systems with different chemical and physical properties will be implemented in the future. These developments are expected to further improve the LiGaMD method for applications in characterizing both ligand binding thermodynamics and kinetics, which should facilitate computer-aided drug design. 


\section{Appendix A Gaussian accelerated molecular dynamics (GaMD)}

Consider a system with $N$ atoms at positions $r \equiv\left\{\vec{r}_{1}, \cdots, \vec{r}_{N}\right\}$. When the system potential $V(r)$ is lower than a reference energy $E$, the modified potential $V^{*}(r)$ of the system is calculated as:

$$
\begin{gathered}
V^{*}(r)=V(r)+\Delta V(r), \\
\Delta V(r)=\left\{\begin{array}{r}
\frac{1}{2} k(E-V(r))^{2}, V(r)<E \\
0, V(r) \geq E
\end{array}\right.
\end{gathered}
$$

where $k$ is the harmonic force constant. The two adjustable parameters $E$ and $k$ are automatically determined based on three enhanced sampling principles ${ }^{25}$. The reference energy needs to be set in the following range:

$$
V_{\max } \leq E \leq V_{\min }+\frac{1}{k}
$$

where $V_{\max }$ and $V_{\min }$ are the system minimum and maximum potential energies. To ensure that Eqn. (A2) is valid, $k$ has to satisfy: $k \leq \frac{1}{V_{\max }-V_{\min }}$ Let us define $k \equiv k_{0} \cdot \frac{1}{V_{\max }-V_{\min }}$, then $0<k_{0} \leq 1$. The standard deviation of $\Delta V$ needs to be small enough (i.e., narrow distribution) to ensure proper energetic reweighting ${ }^{60}: \sigma_{\Delta V}=k\left(E-V_{\text {avg }}\right) \sigma_{V} \leq \sigma_{0}$ where $V_{a v g}$ and $\sigma_{V}$ are the average and standard deviation of the system potential energies, $\sigma_{\Delta V}$ is the standard deviation of $\Delta V$ with $\sigma_{0}$ as a user-specified upper limit (e.g., $10 k_{B} \mathrm{~T}$ ) for proper reweighting. When $E$ is set to the lower bound $E=V_{\max }, k_{0}$ can be calculated as:

$$
k_{0}=\min \left(1.0, k_{0}^{\prime}\right)=\min \left(1.0, \frac{\sigma_{0}}{\sigma_{V}} \cdot \frac{V_{\max }-V_{\min }}{V_{\max }-V_{\text {avg }}}\right) .
$$

Alternatively, when the threshold energy $E$ is set to its upper bound $E=V_{\min }+\frac{1}{k}, k_{0}$ is set to:

$$
k_{0}=k_{0}^{\prime \prime} \equiv\left(1-\frac{\sigma_{0}}{\sigma_{V}}\right) \frac{V_{\max }-V_{\min }}{V_{a v g}-V_{\min }},
$$

if $k_{0}^{\prime \prime}$ is found to be between 0 and 1 . Otherwise, $k_{0}$ is calculated using Eqn. (A3). 
GaMD provides options to boost only the total potential boost (GaMD_Tot), only the dihedral potential energy (GaMD_Dih), both the total and dihedral potential energies (GaMD_Dual), the non-bonded potential energy (GaMD_NB), both the non-bonded potential and dihedral energies (GaMD_Dual_NB), only non-bonded potential energy of the bound ligand (LiGaMD), and both the ligand non-bonded potential energy of the bound ligand and the remaining potential energy of the entire system (LiGaMD_Dual). The dual-boost simulation generally provides higher acceleration than the other single-boost simulations for enhanced sampling. The simulation parameters comprise of settings for calculating the threshold energy values and the effective harmonic force constants of the boost potentials.

\section{Appendix B Energetic reweighting of GaMD simulations}

For energetic reweighting of GaMD simulations to calculate potential of mean force (PMF), the probability distribution along a reaction coordinate is written as $p^{*}(A)$. Given the boost potential $\Delta V(r)$ of each frame, $p^{*}(A)$ can be reweighted to recover the canonical ensemble distribution, $p(A)$, as:

$$
p\left(A_{j}\right)=p^{*}\left(A_{j}\right) \frac{\left\langle e^{\beta \Delta V(r)}\right\rangle_{j}}{\sum_{i=1}^{M}\left\langle p^{*}\left(A_{i}\right) e^{\beta \Delta V(r)\rangle_{i}}\right.}, j=1, \ldots, M,
$$

where $M$ is the number of bins, $\beta=k_{B} T$ and $\left\langle e^{\beta \Delta V(r)}\right\rangle_{j}$ is the ensemble-averaged Boltzmann factor of $\Delta V(r)$ for simulation frames found in the $j^{\text {th }}$ bin. The ensemble-averaged reweighting factor can be approximated using cumulant expansion:

$$
\left\langle e^{\beta \Delta V(r)}\right\rangle=\exp \left\{\sum_{k=1}^{\infty} \frac{\beta^{k}}{k !} C_{k}\right\}
$$

where the first two cumulants are given by: 


$$
\begin{gathered}
C_{1}=\langle\Delta V\rangle, \\
C_{2}=\left\langle\Delta V^{2}\right\rangle-\langle\Delta V\rangle^{2}=\sigma_{v}^{2} .
\end{gathered}
$$

The boost potential obtained from GaMD simulations usually follows near-Gaussian distribution $^{33}$. Cumulant expansion to the second order thus provides a good approximation for computing the reweighting factor ${ }^{25,60}$. The reweighted free energy $F(A)=-k_{B} T \ln p(A)$ is calculated as:

$$
F(A)=F^{*}(A)-\sum_{k=1}^{2} \frac{\beta^{k}}{k !} C_{k}+F_{c}
$$

where $F^{*}(A)=-k_{B} T \ln p^{*}(A)$ is the modified free energy obtained from GaMD simulation and $F_{C}$ is a constant.

\section{Appendix C Reweighting of Biomolecular Kinetics with Kramers' Rate Theory}

A brief summary is provided here for reweighting of biomolecular kinetics from GaMD simulations with Kramers rate theory as described recently ${ }^{26}$. For a particle climbing over potential energy barriers, Kramers showed that the reaction rate depends on temperature and viscosity of the host medium ${ }^{61}$. The reaction rates were derived for both limiting cases of small and large viscosity. In the context of biomolecular simulations in aqueous medium, it is relevant for us to focus on the large viscosity limiting case. Biomolecules move in the high friction ("overdamping”) regime and energy barriers are much greater than $k_{B} T$ ( $k_{B}$ is the Boltzmann's constant and $T$ is temperature). In this case, the reaction rate is calculated as:

$$
k_{R} \cong \frac{2 \pi w_{m} w_{b}}{\xi} e^{-\Delta F / k_{B} T}
$$


where $w_{m}$ and $w_{b}$ are frequencies of the approximated harmonic oscillators (also referred to as curvatures of free energy surface ${ }^{45}$ ) near the energy minimum and barrier, respectively, $\xi$ is the apparent friction coefficient and $\Delta F$ is the free energy barrier of transition.

Without the loss of generality, we consider a 1D potential of mean force (PMF) free energy profile of a reaction coordinate $F(A)$. Near minimum at $A_{m}$, the free energy can be approximated by a harmonic oscillator ${ }^{61}$ of frequency $w_{m}$, i.e., $F(A)=\frac{1}{2}\left(2 \pi w_{m}\right)^{2}\left(A-A_{m}\right)^{2}$. Near barrier at $A_{b}$, the free energy is approximated as $F(A)=F_{b}-\frac{1}{2}\left(2 \pi w_{b}\right)^{2}\left(A-A_{b}\right)^{2}$, where $F_{b}$ is the free energy at $A_{b}$ and $w_{b}$ is the frequency of the approximated harmonic oscillator. Then we can calculate $w_{m}$ and $w_{b}$ as:

$$
w=\sqrt{\frac{\left|F^{\prime \prime}(A)\right|}{2 \pi}}
$$

where $F^{\prime \prime}(A)$ is the second-order derivative of the PMF profile.

The apparent friction coefficient $\xi$ or diffusion coefficient $D$ with $\xi=k_{B} T / D$ can be estimated as follows. First, we calculate a survival function $S(t)$ as the probability that the system remains in an energy well longer than time $t$. In a direct approach ${ }^{46}$, we count the events that the system visits the energy well throughout a simulation. We record and measure the time intervals of each visiting event until the system escapes over an energy barrier. Then we have a time series $T_{i}$, where $i=1,2, \ldots, N$, and $N$ is the total number barrier transitions observed in the simulation. The time series is subsequently ordered such that $\widehat{T}_{1} \leq \widehat{T}_{2} \leq \cdots \leq \widehat{T}_{N}$. With that, the survival function is estimated as $S\left(\widehat{T}_{i}\right) \approx 1-i / N$, which is the probability that the system is trapped in the energy well for time longer than $\widehat{T}_{i}$. Alternatively, we can numerically calculate the timedependent probability density of reaction coordinate $A, \rho(A, t)$ by solving the Smoluchowski equation along 1D PMF profile of the reaction coordinate: 


$$
\frac{\partial \rho(A, t)}{\partial t}=D \frac{\partial}{\partial A}\left[e^{-F(A) / k_{B} T} \frac{\partial}{\partial A}\left(e^{F(A) / k_{B} T} \rho\right)\right]
$$

Then the survival function is calculated as $S(t)=\int_{t}^{\infty} \int_{A_{b 1}}^{A_{b 2}} \rho(A, t) d A d t$, where $A_{b 1}$ and $A_{b 2}$ are two boundaries of the energy well. The initial condition is often set as the Boltzmann distribution of reaction coordinate $A$ in the energy well, i.e., $\rho(A, 0)=e^{-F(A) / k_{B} T}$.

Second, using the above survival functions, we estimate the effective kinetic rates as the negative of the slopes in linear fitting of the $\ln [S(t)]$ versus $t$, i.e., $k=-d \ln [S(t)] / d t$. This is based on the assumption that the survival function exhibits exponential decay as observed in earlier studies $^{46,62}$. Finally, the apparent diffusion coefficient $D$ is obtained by dividing the kinetic rate calculated directly using the transition time series collected from the simulation by that using the probability density solution of the Smoluchowski equation ${ }^{46}$.

The curvatures and energy barriers of the reweighted and modified free energy profiles, as well as the apparent diffusion coefficients, are calculated and used in Kramers' rate equation to determine accelerations of biomolecular kinetics in the GaMD simulations ${ }^{26}$.

\section{Appendix D: Implementation of ligand Gaussian accelerated molecular dynamics}

Ligand Gaussian accelerated molecular dynamics (LiGaMD) is currently implemented in the GPU version of AMBER $20^{63}$, but should be transferable to other molecular dynamics programs as well. LiGaMD provides enhanced sampling of protein-ligand binding and unbinding. Following is a list of the input parameters for a LiGaMD simulation:

igamd Flag to apply boost potential

$=\mathbf{0}$ (default) no boost is applied

$=\mathbf{1}$ boost on the total potential energy only (GaMD_Tot) 
$=\mathbf{2}$ boost on the dihedral energy only (GaMD_Dih)

$=\mathbf{3}$ dual boost on both dihedral and total potential energy (GaMD_Dual)

$=\mathbf{4}$ boost on the non-bonded potential energy only (GaMD_NB)

$=\mathbf{5}$ dual boost on both dihedral and non-bonded potential energy (GaMD_NB_Dual)

$=\mathbf{1 0}$ boost on ligand non-bonded potential energy (LiGaMD)

$=11$ dual boost on both non-bonded potential energy of the bound ligand and the remaining potential energy of the entire system (LiGaMD_Dual)

iE $\quad$ Flag to set the threshold energy $E$ for applying all boost potentials

$=\mathbf{1}$ (default) set the threshold energy to the lower bound $E=V_{\max }$

$=\mathbf{2}$ set the threshold energy to the upper bound $\mathrm{E}=V_{\min }+\left(V_{\max }-V_{\min }\right) / k_{0}$

iEP Flag to overwrite $i E$ and set the threshold energy $E$ for applying the first boost potential in dual-boost schemes

$=\mathbf{1}$ (default) set the threshold energy to the lower bound $E=V_{\max }$

$=\mathbf{2}$ set the threshold energy to the upper bound $\mathrm{E}=V_{\min }+\left(V_{\max }-V_{\min }\right) / k_{0}$

iED Flag to overwrite $i E$ and set the threshold energy $E$ for applying the second boost potential in dual-boost schemes

$=\mathbf{1}$ (default) set the threshold energy to the lower bound $E=V_{\max }$

$=\mathbf{2}$ set the threshold energy to the upper bound $\mathrm{E}=V_{\min }+\left(V_{\max }-V_{\min }\right) / k_{0}$

ntcmdprep The number of preparation conventional molecular dynamics steps. This is used for system equilibration and the potential energies are not collected for calculating their statistics. The default is 200,000 for a simulation with $2 f_{s}$ timestep.

ntcmd The number of initial conventional molecular dynamics simulation steps used to calculate the maximum, minimum, average and standard deviation of the system 
ntebprep The number of preparation biasing molecular dynamics simulation steps. This is used for system equilibration after adding the boost potential and the potential statistics (i.e., $V_{\max }, V_{\min }, V_{\mathrm{avg}}, \sigma_{V}$ ) are not updated during these steps. The default is 200,000 for a simulation with 2 fs timestep.

nteb

potential energies (i.e., $V_{\max }, V_{\min }, V_{\mathrm{avg}}, \sigma_{V}$ ). The default is $1,000,000$ for a simulation with $2 f_{s}$ timestep.

The number of biasing molecular dynamics simulation steps. Potential statistics ( $V_{\max }, V_{\min }, V_{\mathrm{avg}}, \sigma_{V}$ ) are updated between the ntebprep and $\boldsymbol{n t e b}$ steps and used to calculate the GaMD acceleration parameters, particularly $E$ and $k_{0}$. The default is $1,000,000$ for a simulation with $2 f_{s}$ timestep. A greater value may be needed to ensure that the potential statistics and GaMD acceleration parameters level off before running production simulation between the $\boldsymbol{n t e b}$ and $\boldsymbol{n s t l i m}$ (total simulation length) steps. Moreover, nteb can be set to nstlim, by which the potential statistics and GaMD acceleration parameters are updated adaptively throughout the simulation. This in some cases provides more appropriate acceleration.

ntave The number of simulation steps used to calculate the average and standard deviation of potential energies. This variable has already been used in Amber. The default is set to 50,000 for GaMD simulations. It is recommended to be updated as about 4 times of the total number of atoms in the system. Note that ntcmd and nteb need to be multiples of ntave.

irest_gamd Flag to restart GaMD simulation

$=\mathbf{0}$ (default) new simulation. A file "gamd-restart.dat" that stores the maximum, minimum, average and standard deviation of the potential energies needed to 
calculate the boost potentials (depending on the igamd flag) will be saved automatically after GaMD equilibration stage.

$=1$ restart simulation (ntcmd and $n$ teb are set to 0 in this case). The "gamdrestart.dat" file will be read for restart.

sigma0P The upper limit of the standard deviation of the first potential boost that allows for accurate reweighting. The default is 6.0 (unit: $\mathrm{kcal} / \mathrm{mol}$ ).

sigma0D The upper limit of the standard deviation of the second potential boost that allows for accurate reweighting in dual-boost simulations (e.g., igamd = 2, 3, 5 and7). The default is 6.0 (unit: $\mathrm{kcal} / \mathrm{mol}$ ).

timask1 Specifies atoms of the bound ligand in ambmask format. This variable has already been used in Amber. The default is an empty string.

scmask1 Specifies atoms of the bound ligand that will be described using soft core in ambmask format. This variable has already been used in Amber. The default is an empty string.

nlig The total number of ligand molecules in the system. The default is 0 .

ibblig The flag to boost the bound ligand selectively with nlig $>1$

$=\mathbf{0}($ default $)$ no selective boost

$=\mathbf{1}$ boost the bound ligand selectively out of $\boldsymbol{n}$ lig ligand molecules in the system

atom_p Serial number of a protein atom (starting from 1 for the first protein atom) used to calculate the ligand distance. It is used only when $\boldsymbol{i b b l i g}=1$. The default is 0 .

atom_l Serial number of a ligand atom (starting from 1 for the first ligand atom) used to calculate the ligand distance to the protein. It is used only when ibblig $=1$. The default is 0 . 
dblig The cutoff distance between atoms atom $\boldsymbol{p}$ and atom_l for determining whether the ligand is bound in the protein. It is used only when ibblig $=1$. The default is $4.0 \AA$.

Example input parameters used in LiGaMD_Dual simulations of ligand binding to trypsin include the following:

$$
\begin{aligned}
& \text { igamd }=11, \text { irest_gamd }=0, \\
& \text { ntcmd }=700000, \text { nteb }=27300000, \text { ntave }=140000, \\
& \text { ntcmdprep }=280000, \text { ntebprep }=280000, \\
& \text { sigma0P }=4.0, \text { sigma0D }=6.0, \mathrm{iEP}=2, \mathrm{iED}=1, \\
& \text { icfe }=1, \text { ifsc }=1, \text { gti_cpu_output }=0, \text { gti_add_sc }=1, \\
& \text { timask } 1=\text { ':225', scmask1 }=\text { ':225', } \\
& \text { timask2 }=\text { ", scmask } 2=", \\
& \text { ibblig }=1, \text { nlig }=10, \text { atom_p }=2472, \text { atom_1 } 1=4, \mathrm{dblig}=3.7
\end{aligned}
$$

The LiGaMD algorithm is summarized as the following:

\section{LiGaMD \{}

If (irest_gamd $==0$ ) then

For $\mathrm{i}=1, \ldots$, ntcmd // run initial conventional molecular dynamics

If ( $\mathrm{i}>=$ ntcmdprep) Update Vmax, Vmin

If ( $\mathrm{i}>=$ ntcmdprep \& \& i\%ntave $==0)$ Update Vavg, sigmaV

\section{End}

Save Vmax,Vmin,Vavg,sigmaV to "gamd_restart.dat" file

Calc_E_k0(iE,sigma0,Vmax,Vmin,Vavg,sigmaV)

For $\mathrm{i}=\mathrm{ntcmd}+1, \ldots, \mathrm{ntcmd}+\mathrm{nteb} / /$ Run biasing molecular dynamics simulation steps

$$
\begin{aligned}
& \text { delta } \mathrm{V}=0.5 * \mathrm{k} 0 *(\mathrm{E}-\mathrm{V}) * * 2 /(\mathrm{V} \max -\mathrm{Vmin}) \\
& \mathrm{V}=\mathrm{V}+\operatorname{delta} \mathrm{V}
\end{aligned}
$$

If ( $\mathrm{i}>=$ ntcmd + ntebprep) Update Vmax, Vmin

If ( $\mathrm{i}>=$ ntcmd + ntebprep $\& \& \mathrm{i} \%$ ntave $==0$ ) Update Vavg, sigmaV

End

Calc_E_k0(iE,sigma0,Vmax,Vmin,Vavg,sigmaV)

Save Vmax,Vmin,Vavg,sigmaV to "gamd_restart.dat" file

else if (irest_gamd $==1$ ) then

Read Vmax,Vmin,Vavg, sigmaV from "gamd_restart.dat" file

End if

$\operatorname{lig} 0=1 / /$ ID of the bound ligand 
For $\mathrm{i}=$ ntcmd + nteb $+1, \ldots$, nstlim $/ /$ run production simulation

If (ibblig $>0 \& \& \mathrm{i} \%$ ntave $==0$ ) then $/ /$ swap the bound ligand with lig0 for selective boost

For ilig $=1, \ldots$, nlig

$\mathrm{dlig}=\operatorname{distance}($ atom_p, atom_1)

If (dlig $<=$ dblig) blig=ilig

End

If (blig != lig0) Swap atomic coordinates, forces and velocities of ligands blig with lig0 End if

$$
\begin{aligned}
& \text { delta } \mathrm{V}=0.5 * \mathrm{k} 0 *(\mathrm{E}-\mathrm{V}) * * 2 /(\mathrm{Vmax}-\mathrm{Vmin}) \\
& \mathrm{V}=\mathrm{V}+\operatorname{delta} \mathrm{V}
\end{aligned}
$$

End

\}

Subroutine Calc_E_k0(iE,sigma0,Vmax,Vmin,Vavg,sigmaV) \{

if $\mathrm{iE}=1$ :

$$
\begin{aligned}
& \mathrm{E}=\mathrm{Vmax} \\
& \mathrm{k} 0{ }^{\prime}=(\operatorname{sigma} 0 / \text { sigmaV }) *(\mathrm{Vmax}-\mathrm{Vmin}) /(\mathrm{Vmax}-\mathrm{Vavg}) \\
& \mathrm{k} 0=\min \left(1.0, \mathrm{k} 0{ }^{\prime}\right)
\end{aligned}
$$

else if $\mathrm{iE}=2$ :

$\mathrm{k} 0 "=(1-\operatorname{sigma} 0 / \operatorname{sigmaV}) *(\mathrm{Vmax}-\mathrm{Vmin}) /(\mathrm{Vavg}-\mathrm{Vmin})$

if $0<\mathrm{k} 0 "<=1$ :

$$
\mathrm{k} 0=\mathrm{k} 0 "
$$

else

$$
\mathrm{E}=\mathrm{V} \min +(\mathrm{Vmax}-\mathrm{Vmin}) / \mathrm{k} 0
$$

$$
\begin{aligned}
& \mathrm{E}=\mathrm{Vmax} \\
& \mathrm{k} 0^{\prime}=(\operatorname{sigma} 0 / \text { sigmaV }) *(\mathrm{Vmax}-\mathrm{Vmin}) /(\mathrm{Vmax}-\mathrm{Vavg}) \\
& \mathrm{k} 0=\min \left(1.0, \mathrm{k} 0^{\prime}\right)
\end{aligned}
$$

end

end 


\section{Acknowledgements}

We appreciate the help of Prof. David Case for accessing the AMBER git repository to develop our new simulation algorithms. We thank Prof. Chia-en Chang and Dr. Zhiye Tang for kindly sharing molecular dynamics simulation files of the host-guest binding and valuable discussions. We thank Dr. Ferran Feixas for preliminary simulations and valuable discussions on the trypsinbenzamidine system. We also thank Prof. Darrin York and Dr. Taisung Lee for valuable discussions on coding in AMBER. This work used supercomputing resources with allocation award TG-MCB180049 through the Extreme Science and Engineering Discovery Environment (XSEDE), which is supported by National Science Foundation grant number ACI-1548562, and project M2874 through the National Energy Research Scientific Computing Center (NERSC), which is a U.S. Department of Energy Office of Science User Facility operated under Contract No. DE-AC02-05CH11231, and the Research Computing Cluster at the University of Kansas. This work was supported in part by the American Heart Association (Award 17SDG33370094), the National Institutes of Health (R01GM132572) and the startup funding in the College of Liberal Arts and Sciences at the University of Kansas.

\section{Supporting Information}

Five supplementary Tables S1 - S5, seven Figures S1 - S7 and five Movies S1 - S5 are provided in the supporting information. This information is available free of charge via the Internet at http://pubs.acs.org. 


\section{References}

1. Gilson, M. K.; Zhou, H. X., Calculation of protein-ligand binding affinities. Annu Rev Bioph Biom 2007, 36, 21-42.

2. Jorgensen, W. L., The many roles of computation in drug discovery. Science 2004, 303 (5665), 1813-8.

3. Kirkwood, J. G., Statistical Mechanics of Fluid Mixtures. The Journal of Chemical Physics 1935, 3 (5), 300-313.

4. Zwanzig, R. W., High-Temperature Equation of State by a Perturbation Method. I. Nonpolar Gases. The Journal of Chemical Physics 1954, 22 (8), 1420-1426.

5. Gilson, M. K.; Given, J. A.; Bush, B. L.; McCammon, J. A., The statisticalthermodynamic basis for computation of binding affinities: A critical review. Biophysical Journal 1997, 72 (3), 1047-1069.

6. Torrie, G. M.; Valleau, J. P., Nonphysical sampling distributions in Monte Carlo freeenergy estimation: Umbrella sampling. Journal of Computational Physics 1977, 23 (2), 187-199. 7. Villarreal, O. D.; Yu, L.; Rodriguez, R. A.; Chen, L. Y., Computing the binding affinity of a ligand buried deep inside a protein with the hybrid steered molecular dynamics. Biochem Biophys Res Commun 2017, 483 (1), 203-208.

8. Limongelli, V.; Bonomi, M.; Parrinello, M., Funnel metadynamics as accurate binding free-energy method. Proc Natl Acad Sci 2013, 110 (16), 6358-63.

9. Srinivasan, J.; Cheatham, T. E.; Cieplak, P.; Kollman, P. A.; Case, D. A., Continuum Solvent Studies of the Stability of DNA, RNA, and Phosphoramidate-DNA Helices. J Am Chem Soc 1998, 120 (37), 9401-9409.

10. (a) Yin, J.; Henriksen, N. M.; Slochower, D. R.; Shirts, M. R.; Chiu, M. W.; Mobley, D. L.; Gilson, M. K., Overview of the SAMPL5 host-guest challenge: Are we doing better? $J$ Comput Aided Mol Des 2017, 31 (1), 1-19; (b) Muddana, H. S.; Fenley, A. T.; Mobley, D. L.; Gilson, M. K., The SAMPL4 host-guest blind prediction challenge: an overview. J Comput Aided Mol Des 2014, 28 (4), 305-17; (c) Muddana, H. S.; Varnado, C. D.; Bielawski, C. W.; Urbach, A. R.; Isaacs, L.; Geballe, M. T.; Gilson, M. K., Blind prediction of host-guest binding affinities: a new SAMPL3 challenge. J Comput Aided Mol Des 2012, 26 (5), 475-87.

11. (a) Gaieb, Z.; Parks, C. D.; Chiu, M.; Yang, H.; Shao, C.; Walters, W. P.; Lambert, M. H.; Nevins, N.; Bembenek, S. D.; Ameriks, M. K.; Mirzadegan, T.; Burley, S. K.; Amaro, R. E.; Gilson, M. K., D3R Grand Challenge 3: blind prediction of protein-ligand poses and affinity rankings. J Comput Aided Mol Des 2019; (b) Gathiaka, S.; Liu, S.; Chiu, M.; Yang, H.; Stuckey, J. A.; Kang, Y. N.; Delproposto, J.; Kubish, G.; Dunbar, J. B., Jr.; Carlson, H. A.; Burley, S. K.; Walters, W. P.; Amaro, R. E.; Feher, V. A.; Gilson, M. K., D3R grand challenge 2015: Evaluation of protein-ligand pose and affinity predictions. J Comput Aided Mol Des 2016, 30 (9), 651-668; (c) Gaieb, Z.; Liu, S.; Gathiaka, S.; Chiu, M.; Yang, H.; Shao, C.; Feher, V. A.; Walters, W. P.; Kuhn, B.; Rudolph, M. G.; Burley, S. K.; Gilson, M. K.; Amaro, R. E., D3R Grand Challenge 2: blind prediction of protein-ligand poses, affinity rankings, and relative binding free energies. J Comput Aided Mol Des 2018, 32 (1), 1-20.

12. (a) Schuetz, D. A.; de Witte, W. E. A.; Wong, Y. C.; Knasmueller, B.; Richter, L.; Kokh, D. B.; Sadiq, S. K.; Bosma, R.; Nederpelt, I.; Heitman, L. H.; Segala, E.; Amaral, M.; Guo, D.; Andres, D.; Georgi, V.; Stoddart, L. A.; Hill, S.; Cooke, R. M.; De Graaf, C.; Leurs, R.; Frech, M.; Wade, R. C.; de Lange, E. C. M.; IJzerman, A. P.; Muller-Fahrnow, A.; Ecker, G. F., 
Kinetics for Drug Discovery: an industry-driven effort to target drug residence time. Drug Discov Today 2017, 22 (6), 896-911; (b) Tonge, P. J., Drug-Target Kinetics in Drug Discovery. ACS chemical neuroscience 2018, 9 (1), 29-39.

13. (a) Dror, R. O.; Pan, A. C.; Arlow, D. H.; Borhani, D. W.; Maragakis, P.; Shan, Y.; Xu, H.; Shaw, D. E., Pathway and mechanism of drug binding to G-protein-coupled receptors. Proc Natl Acad Sci 2011, 108 (32), 13118-23; (b) Shan, Y.; Kim, E. T.; Eastwood, M. P.; Dror, R. O.; Seeliger, M. A.; Shaw, D. E., How does a drug molecule find its target binding site? J Am Chem Soc 2011, 133 (24), 9181-3.

14. Buch, I.; Giorgino, T.; De Fabritiis, G., Complete reconstruction of an enzyme-inhibitor binding process by molecular dynamics simulations. Proc. Natl. Acad. Sci. U. S. A. 2011, 108 (25), 10184-10189.

15. (a) Kappel, K.; Miao, Y.; McCammon, J. A., Accelerated Molecular Dynamics Simulations of Ligand Binding to a Muscarinic G-protein Coupled Receptor. Quarterly Reviews of Biophysics 2015, 48 (04), 479-487; (b) Saglam, A. S.; Chong, L. T., Protein-protein binding pathways and calculations of rate constants using fully-continuous, explicit-solvent simulations. Chemical Science 2019, 10 (8), 2360-2372.

16. (a) Tiwary, P.; Limongelli, V.; Salvalaglio, M.; Parrinello, M., Kinetics of protein-ligand unbinding: Predicting pathways, rates, and rate-limiting steps. Proc Natl Acad Sci 2015, 112 (5), E386-E391; (b) Casasnovas, R.; Limongelli, V.; Tiwary, P.; Carloni, P.; Parrinello, M., Unbinding Kinetics of a p38 MAP Kinase Type II Inhibitor from Metadynamics Simulations. $J$ Am Chem Soc 2017, 139 (13), 4780-4788.

17. (a) Kokh, D. B.; Amaral, M.; Bomke, J.; Gradler, U.; Musil, D.; Buchstaller, H. P.; Dreyer, M. K.; Frech, M.; Lowinski, M.; Vallee, F.; Bianciotto, M.; Rak, A.; Wade, R. C., Estimation of Drug-Target Residence Times by tau-Random Acceleration Molecular Dynamics Simulations. J Chem Theory Comput 2018, 14 (7), 3859-3869; (b) Wang, T.; Duan, Y., Chromophore channeling in the G-protein coupled receptor rhodopsin. J. Am. Chem. Soc. 2007, 129 (22), 6970-6971.

18. (a) Park, S.; Khalili-Araghi, F.; Tajkhorshid, E.; Schulten, K., Free energy calculation from steered molecular dynamics simulations using Jarzynski's equality. J Chem Phys 2003, 119 (6), 3559-3566; (b) Gonzalez, A.; Perez-Acle, T.; Pardo, L.; Deupi, X., Molecular Basis of Ligand Dissociation in beta-Adrenergic Receptors. PLoS One 2011, 6 (9), e23815.

19. Dickson, A.; Lotz, S. D., Multiple Ligand Unbinding Pathways and Ligand-Induced Destabilization Revealed by WExplore. Biophys $J$ 2017, 112 (4), 620-629.

20. Deb, I.; Frank, A. T., Accelerating Rare Dissociative Processes in Biomolecules Using Selectively Scaled MD Simulations. J Chem Theory Comput 2019, 15 (11), 5817-5828.

21. Tang, Z. Y.; Chang, C. E. A., Binding Thermodynamics and Kinetics Calculations Using Chemical Host and Guest: A Comprehensive Picture of Molecular Recognition. J Chem Theory Comput 2018, 14 (1), 303-318.

22. Plattner, N.; Noe, F., Protein conformational plasticity and complex ligand-binding kinetics explored by atomistic simulations and Markov models. Nat Commun 2015, 6, 7653.

23. Tiwary, P.; Parrinello, M., From Metadynamics to Dynamics. Phys Rev Lett 2013, 111 (23).

24. Shao, Q.; Zhu, W., Exploring the Ligand Binding/Unbinding Pathway by Selectively Enhanced Sampling of Ligand in a Protein-Ligand Complex. J Phys Chem B 2019, 123 (38), 7974-7983. 
25. Miao, Y.; Feher, V. A.; McCammon, J. A., Gaussian Accelerated Molecular Dynamics: Unconstrained Enhanced Sampling and Free Energy Calculation. J Chem Theory Comput 2015, $11(8), 3584-3595$.

26. Miao, Y., Acceleration of Biomolecular Kinetics in Gaussian Accelerated Molecular Dynamics. J Chem Phys 2018, 149 (7), 072308.

27. Miao, Y.; McCammon, J. A., Unconstrained enhanced sampling for free energy calculations of biomolecules: a review. Molecular simulation 2016, 42 (13), 1046-1055.

28. (a) Hamelberg, D.; Mongan, J.; McCammon, J. A., Accelerated molecular dynamics: A promising and efficient simulation method for biomolecules. J Chem Phys 2004, 120 (24), 11919-11929; (b) Voter, A. F., Hyperdynamics: Accelerated molecular dynamics of infrequent events. Physical Review Letters 1997, 78 (20), 3908.

29. Shen, T. Y.; Hamelberg, D., A statistical analysis of the precision of reweighting-based simulations. J Chem Phys 2008, 129 (3), 034103.

30. Case, D.; Babin, V.; Berryman, J.; Betz, R.; Cai, Q.; Cerutti, D.; Cheatham III, T.; Darden, T.; Duke, R.; Gohlke, H., Amber 14, University of California, San Francisco. 2014. 31. Pang, Y. T.; Miao, Y.; Wang, Y.; McCammon, J. A., Gaussian Accelerated Molecular Dynamics in NAMD. J Chem Theory Comput 2017, 13 (1), 9-19.

32. Oshima, H.; Re, S.; Sugita, Y., Replica-Exchange Umbrella Sampling Combined with Gaussian Accelerated Molecular Dynamics for Free-Energy Calculation of Biomolecules. $J$ Chem Theory Comput 2019, 15 (10), 5199-5208.

33. (a) Miao, Y.; McCammon, J. A., Gaussian Accelerated Molecular Dynamics: Theory, Implementation and Applications. Annual Reports in Computational Chemistry 2017, 13, 231278; (b) Pawnikar, S. P.; Miao, Y., Pathway and Mechanism of Drug Binding to Chemokine Receptors Revealed by Accelerated Molecular Simulations. Future Med Chem 2020, In Press. 34. (a) Wang, J.; Miao, Y., Mechanistic Insights into Specific G Protein Interactions with Adenosine Receptors. The Journal of Physical Chemistry B 2019, 123 (30), 6462-6473; (b) Miao, Y.; McCammon, J. A., Mechanism of the G-Protein Mimetic Nanobody Binding to a Muscarinic G-Protein-Coupled Receptor. Proc Natl Acad Sci U S A 2018, 115 (12), 3036-3041. 35. Bhattarai, A.; Wang, J.; Miao, Y., G-Protein-Coupled Receptor-Membrane Interactions Depend on the Receptor Activation State. J Comput Chem 2019, 41, 460-471.

36. (a) East, K. W.; Newton, J. C.; Morzan, U. N.; Narkhede, Y. B.; Acharya, A.; Skeens, E.; Jogl, G.; Batista, V. S.; Palermo, G.; Lisi, G. P., Allosteric Motions of the CRISPR-Cas9 HNH Nuclease Probed by NMR and Molecular Dynamics. J Am Chem Soc 2020, 142 (3), 1348-1358; (b) Ricci, C. G.; Chen, J. S.; Miao, Y.; Jinek, M.; Doudna, J. A.; McCammon, J. A.; Palermo, G., Deciphering Off-Target Effects in CRISPR-Cas9 through Accelerated Molecular Dynamics. ACS Central Science 2019, 5 (4), 651-662.

37. Wereszczynski, J.; McCammon, J. A., Using Selectively Applied Accelerated Molecular Dynamics to Enhance Free Energy Calculations. J Chem Theory Comput 2010, 6 (11), 3285 3292.

38. (a) Zheng, L. Q.; Yang, W., Essential energy space random walks to accelerate molecular dynamics simulations: Convergence improvements via an adaptive-length self-healing strategy. $J$ Chem Phys 2008, 129 (1), 014105; (b) Li, H.; Min, D.; Liu, Y.; Yang, W., Essential energy space random walk via energy space metadynamics method to accelerate molecular dynamics simulations. J Chem Phys 2007, 127 (9), 094101. 
39. Liu, P.; Kim, B.; Friesner, R. A.; Berne, B. J., Replica exchange with solute tempering: A method for sampling biological systems in explicit water. Proc Natl Acad Sci 2005, 102 (39), 13749-13754.

40. Wang, L.; Friesner, R. A.; Berne, B. J., Replica exchange with solute scaling: a more efficient version of replica exchange with solute tempering (REST2). J Phys Chem B 2011, 115 (30), 9431-8.

41. (a) Vanommeslaeghe, K.; MacKerell, A. D., Jr., CHARMM additive and polarizable force fields for biophysics and computer-aided drug design. Biochimica et biophysica acta 2014; (b) Duan, Y.; Wu, C.; Chowdhury, S.; Lee, M. C.; Xiong, G. M.; Zhang, W.; Yang, R.; Cieplak, P.; Luo, R.; Lee, T.; Caldwell, J.; Wang, J. M.; Kollman, P., A point-charge force field for molecular mechanics simulations of proteins based on condensed-phase quantum mechanical calculations. J Comput Chem 2003, 24 (16), 1999-2012.

42. Miao, Y.; Bhattarai, A.; Nguyen, A. T. N.; Christopoulos, A.; May, L. T., Structural Basis for Binding of Allosteric Drug Leads in the Adenosine A1 Receptor. Sci. Rep. 2018, 8 (1), 16836.

43. (a) Doudou, S.; Burton, N. A.; Henchman, R. H., Standard Free Energy of Binding from a One-Dimensional Potential of Mean Force. J Chem Theory Comput 2009, 5 (4), 909-18; (b) Buch, I.; Giorgino, T.; De Fabritiis, G., Complete reconstruction of an enzyme-inhibitor binding process by molecular dynamics simulations. Proc Natl Acad Sci 2011, 108 (25), 10184-10189. 44. Ferruz, N.; De Fabritiis, G., Binding Kinetics in Drug Discovery. Mol Inform 2016, 35 (6-7), 216-26.

45. (a) Doshi, U.; Hamelberg, D., Extracting Realistic Kinetics of Rare Activated Processes from Accelerated Molecular Dynamics Using Kramers' Theory. J Chem Theory Comput 2011, 7 (3), 575-581; (b) Frank, A. T.; Andricioaei, I., Reaction Coordinate-Free Approach to Recovering Kinetics from Potential-Scaled Simulations: Application of Kramers' Rate Theory. The Journal of Physical Chemistry B 2016, 120 (33), 8600-8605.

46. Hamelberg, D.; Shen, T.; McCammon, J. A., Relating kinetic rates and local energetic roughness by accelerated molecular-dynamics simulations - art. no. 2411003. J. Chem. Phys. 2005, 122 (24).

47. Truhlar, D. G.; Garrett, B. C.; Klippenstein, S. J., Current Status of Transition-State Theory. The Journal of Physical Chemistry 1996, 100 (31), 12771-12800.

48. Humphrey, W.; Dalke, A.; Schulten, K., VMD: Visual molecular dynamics. Journal of Molecular Graphics \& Modelling 1996, 14 (1), 33-38.

49. Roe, D. R.; Cheatham, T. E., PTRAJ and CPPTRAJ: Software for Processing and Analysis of Molecular Dynamics Trajectory Data. J Chem Theory Comput 2013, 9 (7), 30843095.

50. Marquart, M.; Walter, J.; Deisenhofer, J.; Bode, W.; Huber, R., The geometry of the reactive site and of the peptide groups in trypsin, trypsinogen and its complexes with inhibitors. Acta Crystallographica Section B 1983, 39 (4), 480-490.

51. Case, D. A.; Cheatham, T. E.; Darden, T.; Gohlke, H.; Luo, R.; Merz, K. M.; Onufriev, A.; Simmerling, C.; Wang, B.; Woods, R. J., The Amber biomolecular simulation programs. $J$ Comput Chem 2005, 26 (16), 1668-1688.

52. Wang, J.; Wolf, R. M.; Caldwell, J. W.; Kollman, P. A.; Case, D. A., Development and testing of a general amber force field. Journal of computational chemistry 2004, 25 (9), 11571174. 
53. Maier, J. A.; Martinez, C.; Kasavajhala, K.; Wickstrom, L.; Hauser, K. E.; Simmerling, C., ff14SB: Improving the Accuracy of Protein Side Chain and Backbone Parameters from ff99SB. Journal of Chemical Theory and Computation 2015, 11 (8), 3696-3713.

54. Cieplak, P.; Cornell, W. D.; Bayly, C.; Kollman, P. A., Application of the multimolecule and multiconformational RESP methodology to biopolymers: charge derivation for DNA, RNA, and proteins. Journal of Computational Chemistry 1995, 16 (11), 1357-1377.

55. Jorgensen, W. L.; Chandrasekhar, J.; Madura, J. D.; Impey, R. W.; Klein, M. L., Comparison of Simple Potential Functions for Simulating Liquid Water. J Chem Phys 1983, 79 (2), 926-935.

56. $\quad$ Ester, M.; Kriegel, H.-P.; Sander, J.; Xu, X., A density-based algorithm for discovering clusters in large spatial databases with noise. Knowledge Discovery and Data Mining 1996, 96 (34), 226-231.

57. Voet, D.; Voet, J. G.; Pratt, C. W., Fundamentals of Biochemistry, 4th ed. Ch. 11, 339349.

58. Guillain, F.; Thusius, D., The use of proflavin as an indicator in temperature-jump studies of the binding of a competitive inhibitor to trypsin. J Am Chem Soc 1970, 92 (18), 5534-6.

59. Wade, R. C.; Gabdoulline, R. R.; Ludemann, S. K.; Lounnas, V., Electrostatic steering and ionic tethering in enzyme-ligand binding: insights from simulations. Proc Natl Acad Sci 1998, 95 (11), 5942-9.

60. Miao, Y.; Sinko, W.; Pierce, L.; Bucher, D.; McCammon, J. A., Improved reweighting of accelerated molecular dynamics simulations for free energy calculation. J Chem Theory Comput 2014, 10 (7), 2677-2689.

61. Kramers, H. A., Brownian motion in a field of force and the diffusion model of chemical reactions. Physica 1940, 7, 284-304.

62. Xin, Y.; Doshi, U.; Hamelberg, D., Examining the limits of time reweighting and Kramers' rate theory to obtain correct kinetics from accelerated molecular dynamics. J Chem Phys 2010, 132 (22), 224101.

63. Le Grand, S.; Gotz, A. W.; Walker, R. C., SPFP: Speed without compromise-A mixed precision model for GPU accelerated molecular dynamics simulations. Comput. Phys. Commun. 2013, 184 (2), 374-380. 
Table 1 Summary of host-guest binding thermodynamics and kinetics obtained from GaMD simulations on the binding of two guest molecules (aspirin and 1-butanol) to the $\beta$-cyclodextrin (CD) host. The CD host is modeled with the GAFF and q4MD force fields. $\Delta V$ is the system boost potential applied in GaMD simulations. $\Delta G$ is the ligand binding free energy. $N_{D}$ and $N_{B}$ are the number of host-guest dissociation and binding events collected from the individual simulations. $k_{o n} *$ and $k_{o f f} *$ are the kinetic dissociation and binding rate constants, which are calculated from only the GaMD simulations with $N_{D}>0$ and $N_{B}>0$.

\begin{tabular}{|c|c|c|c|c|c|c|c|c|}
\hline Host & Ligand & $\begin{array}{c}\text { GaMD } \\
(300 \mathrm{~ns} \times 3) \\
\end{array}$ & $\begin{array}{c}\Delta V \\
\text { (kcal } / \mathrm{mol})\end{array}$ & $N_{D}$ & $N_{B}$ & $\begin{array}{c}\Delta G \\
(\mathrm{kcal} / \mathrm{mol})\end{array}$ & $\begin{array}{c}k_{o n}{ }^{*} \\
\left(\times 10^{8} \mathrm{M}^{-1} \cdot \mathrm{s}^{-1}\right)\end{array}$ & $\begin{array}{c}k_{o f f} * \\
\left(\times 10^{6} \mathrm{~s}^{-1}\right)\end{array}$ \\
\hline \multirow{8}{*}{$\begin{array}{l}\text { CD: } \\
\text { GAFF }\end{array}$} & \multirow{6}{*}{ Aspirin } & GaMD_Tot & $6.48 \pm 2.59$ & $0,2,1$ & $0,1,0$ & - & - & - \\
\hline & & GaMD_Dual & $10.37 \pm 2.98$ & $0,3,0$ & $0,3,0$ & - & - & - \\
\hline & & GaMD_NB & $4.15 \pm 2.26$ & $1,1,2$ & $1,0,1$ & - & - & - \\
\hline & & GaMD_NB_Dual & $8.75 \pm 2.82$ & $0,1,0$ & $0,1,0$ & - & - & - \\
\hline & & LiGaMD & $2.60 \pm 1.27$ & $3,1,2$ & $2,1,1$ & $-4.90 \pm 0.03$ & $3.05 \pm 0.92$ & $40.67 \pm 24.25$ \\
\hline & & LiGaMD_Dual & $9.18 \pm 2.84$ & $2,4,2$ & $2,3,2$ & $-2.89 \pm 0.18$ & $3.93 \pm 0.98$ & $275.6 \pm 57.5$ \\
\hline & \multirow{2}{*}{$\begin{array}{c}1- \\
\text { Butanol } \\
\end{array}$} & LiGaMD & $2.54 \pm 1.00$ & $1,0,0$ & $1,0,0$ & - & - & - \\
\hline & & LiGaMD_Dual & $9.51 \pm 2.85$ & $1,0,1$ & $1,0,1$ & - & - & - \\
\hline \multirow{8}{*}{$\begin{array}{c}\text { CD: } \\
\text { q4MD }\end{array}$} & \multirow{6}{*}{ Aspirin } & GaMD_Tot & $6.22 \pm 2.52$ & $0,2,0$ & $0,2,0$ & - & - & - \\
\hline & & GaMD_Dual & $10.77 \pm 3.02$ & $1,0,0$ & $1,0,0$ & - & - & - \\
\hline & & GaMD_NB & $4.13 \pm 2.17$ & $0,2,2$ & $0,2,2$ & - & - & - \\
\hline & & GaMD_NB_Dual & $8.78 \pm 2.83$ & $0,2,2$ & $0,2,2$ & - & - & - \\
\hline & & LiGaMD & $2.84 \pm 1.34$ & $4,3,2$ & $3,2,2$ & $-4.74 \pm 0.53$ & $8.99 \pm 5.02$ & $42.82 \pm 15.41$ \\
\hline & & LiGaMD_Dual & $10.06 \pm 3.15$ & $8,7,4$ & $8,6,3$ & $-3.34 \pm 0.15$ & $10.37 \pm 2.60$ & $56.61 \pm 19.05$ \\
\hline & \multirow{2}{*}{$\begin{array}{c}1- \\
\text { Butanol } \\
\end{array}$} & LiGaMD & $2.87 \pm 1.12$ & $9,4,7$ & $9,4,7$ & $-2.84 \pm 0.58$ & $8.76 \pm 2.93$ & $482.8 \pm 246.3$ \\
\hline & & LiGaMD_Dual & $9.74 \pm 2.93$ & $9,13,8$ & $9,13,8$ & $-1.34 \pm 0.10$ & $13.53 \pm 2.15$ & $436.8 \pm 90.7$ \\
\hline
\end{tabular}


Table 2 Comparison of the host-guest binding free energies calculated from the LiGaMD simulations, LiGaMD_Dual simulations, cMD simulations ${ }^{21}$ and experimental data ${ }^{21}$.

\begin{tabular}{|c|c|c|c|c|c|}
\hline \multirow[b]{2}{*}{ Host } & \multirow[b]{2}{*}{ Ligand } & \multicolumn{4}{|c|}{$\Delta G_{\text {sim }}-\Delta G_{\exp }(\mathrm{kcal} / \mathrm{mol})$} \\
\hline & & $\begin{array}{c}\mathrm{cMD} \\
\left(\Delta G_{\text {compl } 1}\right)\end{array}$ & $\begin{array}{c}\mathrm{cMD} \\
\left(\Delta G_{\text {comp } 2)}\right.\end{array}$ & LiGaMD & LiGaMD_Dual \\
\hline $\begin{array}{c}\text { CD: } \\
\text { GAFF }\end{array}$ & Aspirin & $-0.10 \pm 0.35$ & $1.47 \pm 0.06$ & $-1.16 \pm 0.03$ & $0.85 \pm 0.18$ \\
\hline \multirow{2}{*}{$\begin{array}{c}\text { CD: } \\
\text { q4MD }\end{array}$} & Aspirin & $-2.59 \pm 0.37$ & $-0.37 \pm 0.05$ & $-1.00 \pm 0.53$ & $0.40 \pm 0.15$ \\
\hline & 1-Butanol & $0.34 \pm 0.39$ & $-0.60 \pm 0.19$ & $-1.17 \pm 0.61$ & $0.33 \pm 0.21$ \\
\hline
\end{tabular}


Table 3 Accelerations of host-guest dissociation and binding rate constants obtained from (A) LiGaMD and (B) LiGaMD_Dual simulations as compared directly with previous cMD simulations $^{\mathbf{2 1}}$ and those derived using the Transition State Theory and Kramers' Rate Theory.

\begin{tabular}{|c|c|c|c|c|c|c|c|}
\hline \multirow{2}{*}{ Host } & \multirow{2}{*}{ Ligand } & \multicolumn{2}{|c|}{ LiGaMD vs. cMD } & \multicolumn{2}{c|}{ Transition State Theory } & \multicolumn{2}{c|}{ Kramer's Rate Theory } \\
\cline { 3 - 8 } & $k_{o n} * / k_{o n}$ & $k_{o f f} * / k_{o f f}$ & $k_{o n} * / k_{o n}$ & $k_{o f f} * / k_{o f f}$ & $k_{o n} * / k_{o n}$ & $k_{o f f} * / k_{o f f}$ \\
\hline $\begin{array}{c}\text { CD: } \\
\text { GAFF }\end{array}$ & Aspirin & $0.28 \pm 0.08$ & $1.69 \pm 1.03$ & $0.12 \pm 0.09$ & $15.07 \pm 12.94$ & $0.20 \pm 0.16$ & $12.19 \pm 10.47$ \\
\hline $\begin{array}{c}\text { CD: } \\
\text { q4MD }\end{array}$ & Aspirin & $0.28 \pm 0.16$ & $13.81 \pm 6.39$ & $0.19 \pm 0.16$ & $21.59 \pm 16.11$ & $0.02 \pm 0.02$ & $12.36 \pm 9.22$ \\
\cline { 2 - 8 } & 1-Butanol & $0.58 \pm 0.20$ & $14.63 \pm 7.47$ & $0.43 \pm 0.34$ & $8.77 \pm 6.72$ & $0.55 \pm 0.43$ & $7.91 \pm 6.06$ \\
\hline
\end{tabular}

(A) LiGaMD simulations

\begin{tabular}{|c|c|c|c|c|c|c|c|}
\hline \multirow{2}{*}{ Host } & \multirow{2}{*}{ Ligand } & \multicolumn{2}{|c|}{ LiGaMD_Dual vs. cMD } & \multicolumn{2}{c|}{ Transition State Theory } & \multicolumn{2}{c|}{ Kramer's Rate Theory } \\
\cline { 3 - 8 } & $k_{o n} * / k_{o n}$ & $k_{o f f} * / k_{\text {off }}$ & $k_{o n} * / k_{o n}$ & $k_{\text {off }} * / k_{\text {off }}$ & $k_{o n} * / k_{\text {on }}$ & $k_{\text {off }} * / k_{\text {off }}$ \\
\hline $\begin{array}{c}\text { CD: } \\
\text { GAFF }\end{array}$ & Aspirin & $0.36 \pm 0.09$ & $11.48 \pm 2.79$ & $0.09 \pm 0.07$ & $11.89 \pm 9.36$ & $0.06 \pm 0.05$ & $7.97 \pm 6.27$ \\
\hline $\begin{array}{c}\text { CD: } \\
\text { q4MD }\end{array}$ & Aspirin & $0.32 \pm 0.09$ & $18.26 \pm 8.12$ & $0.18 \pm 0.14$ & $41.37 \pm 22.17$ & $0.23 \pm 0.18$ & $22.82 \pm 12.23$ \\
\cline { 2 - 8 } & 1-Butanol & $0.90 \pm 0.14$ & $13.24 \pm 2.76$ & $0.23 \pm 0.11$ & $5.54 \pm 2.73$ & $1.03 \pm 0.47$ & $5.96 \pm 2.94$ \\
\hline
\end{tabular}

(B) LiGaMD_Dual simulations 
Table 4 Summary of LiGaMD_Dual production simulations performed on the benzamidine ligand binding to trypsin. $\Delta V$ is the GaMD boost potential. $N_{D}$ and $N_{B}$ are the number of host-guest dissociation and binding events recorded from individual simulations.

\begin{tabular}{|c|c|c|c|c|c|c|}
\hline System & Method & ID & $\begin{array}{c}\text { Length } \\
(\mathrm{ns})\end{array}$ & $\Delta V(\mathrm{kcal} / \mathrm{mol})$ & $N_{D}$ & $N_{B}$ \\
\hline \multirow{3}{*}{$\begin{array}{c}\text { Trypsin - } \\
\text { benzamidine }\end{array}$} & \multirow{2}{*}{ LiGaMD_Dual } & Sim1 & 1000 & $20.93 \pm 4.24$ & 3 & 3 \\
\cline { 3 - 7 } & & Sim2 & 1000 & $21.64 \pm 4.67$ & 5 & 4 \\
\cline { 3 - 7 } & Sim3 & 1000 & $20.99 \pm 4.23$ & 11 & 10 \\
\cline { 3 - 7 } & Sim4 & 1000 & $20.87 \pm 4.18$ & 4 & 4 \\
\cline { 2 - 6 } & Sim5 & 1000 & $20.94 \pm 4.18$ & 4 & 4 \\
\hline
\end{tabular}


Table 5 Comparison of trypsin protein-ligand binding free energy and kinetic rates obtained from experimental data and LiGaMD_Dual simulations. $\Delta G$ is the ligand binding free energy. $k_{\text {on }}$ and $k_{\text {off }}$ are the kinetic dissociation and binding rate constants, respectively, from experimental data or LiGaMD_Dual simulations with reweighting using Kramers' rate theory. $k_{o n} *$ and $k_{\text {off }} *$ are the accelerated kinetic dissociation and binding rate constants calculated directly from LiGaMD_Dual simulations without reweighting.

\begin{tabular}{|c|c|c|c|c|c|}
\hline \multicolumn{1}{|c|}{ Method } & $\begin{array}{c}\Delta G \\
(\mathrm{kcal} / \mathrm{mol})\end{array}$ & $\begin{array}{c}k_{\text {on }} \\
\left(\mathrm{M}^{-1} \cdot \mathrm{s}^{-1}\right)\end{array}$ & $\begin{array}{c}k_{\text {off }} \\
\left(\mathrm{s}^{-1}\right)\end{array}$ & $\begin{array}{c}k_{\text {on }}^{*} \\
\left(\mathrm{M}^{-1} \cdot \mathrm{s}^{-1}\right)\end{array}$ & $\begin{array}{c}k_{\text {off }}{ }^{*} \\
\left(\mathrm{~s}^{-1}\right)\end{array}$ \\
\hline Experiment & -6.2 & $2.9 \times 10^{7}$ & $600 \pm 300$ & - & - \\
\hline LiGaMD_Dual & $-6.13 \pm 0.35$ & $1.15 \pm 0.79 \times 10^{7}$ & $3.53 \pm 1.41$ & $1.58 \pm 1.09 \times 10^{8}$ & $3.40 \pm 1.36 \times 10^{7}$ \\
\hline
\end{tabular}




\section{Figure Captions}

Fig. 1 Comparison of potential of mean force (PMF) free energy profiles calculated from conventional MD (cMD), LiGaMD and dual-boost LiGaMD (LiGaMD_Dual) simulations of hostguest binding: (A-B) Computational models of the $\beta$-cyclodextrin (CD) host (balls-and-sticks) in the presence of guest (A) aspirin and (B) 1-butanol (thick sticks) in aqueous medium (cyan). (CF) PMF profiles calculated from microsecond-timescale cMD simulations and three independent 300 ns LiGaMD and LiGaMD_Dual simulations of (C) CD using the GAFF force field with aspirin, (D) CD using the GAFF force field with 1-butanol, (E) CD using the q4MD force field with aspirin, (F) CD using the q4MD force field with 1-butanol.

Fig. 2 Free energy profiles and low-energy conformational states of guest binding to the CD host that was modeled with the GAFF force field: (A) PMF profiles of the host radius of gyration $\left(R_{g}\right)$ calculated from cMD simulations in the ligand-free (apo), aspirin and 1-butanol binding forms. Two low-energy states ("Compact" and "Open") are identified for the CD host. (B) Three representative conformational states observed in simulations of guest aspirin binding to CD modeled with GAFF: the "Bound (B)", "Intermediate (I)" and "Unbound (U)", in which the CD host adopted primarily the Open, Compact and Compact conformations, respectively. (C-E) 2D PMF profiles of the host $R_{g}$ versus the center-of-mass distance between host-guest of (C) aspirin binding from $9500 \mathrm{~ns}$ cMD simulation, (D) aspirin binding from three $300 \mathrm{~ns}$ LiGaMD_Dual simulation combined, (E) 1-butanol binding from $6500 \mathrm{~ns}$ cMD simulation, and (F) 1-butanol binding from three 300 ns LiGaMD_Dual simulation combined. The low-energy states are labeled. Fig. 3 Free energy profiles and low-energy conformational states of guest binding to the CD host that was modeled with the q4MD force field: (A) PMF profiles of the host radius of gyration $\left(R_{g}\right)$ calculated from cMD simulations in the ligand-free (apo), aspirin and 1-butanol binding forms. 
Two low-energy states ("Compact" and "Open") are labeled for the CD host. (B) Three representative conformational states observed in simulations of aspirin binding to CD modeled with q4MD: the "Bound (B)", "Intermediate (I)" and "Unbound (U)", in which the CD host all adopted primarily the Open conformation. (C-E) 2D PMF profiles regarding the host $R_{g}$ versus center-of-mass distance between host-guest of (C) aspirin binding from $6000 \mathrm{~ns}$ cMD simulation, (D) aspirin binding from three $300 \mathrm{~ns}$ LiGaMD_Dual simulation combined, (E) 1-butanol binding from $5000 \mathrm{~ns}$ cMD simulation, and (F) 1-butanol binding from three $300 \mathrm{~ns}$ LiGaMD_Dual simulation combined. The low-energy states are labeled.

Fig. 4 (A-F) The original (reweighted) and modified (no reweighting) PMF profiles of (A) LiGaMD simulations of CD using the GAFF force field with aspirin, (B) LiGaMD_Dual simulations of CD using the GAFF force field with aspirin, (C) LiGaMD simulations of CD using the q4MD force field with aspirin, (D) LiGaMD_Dual simulations of CD using the q4MD force field with aspirin, (E) LiGaMD simulations of CD using the q4MD force field with 1-butanol, and (F) LiGaMD_Dual simulations of CD using the q4MD force field with 1-butanol.

Fig. 5 LiGaMD_Dual simulations have captured repetitive binding and unbinding of the benzamidine ligand in the trypsin protein: (A) time courses of distances between the $\mathrm{N}$ atom in benzamidine and CG atom of Asp189 in trypsin calculated from a representative $1 \mu$ s LiGaMD_Dual simulation (Sim2 in Table 5). Distance plots of the other simulations are provided in Figure S5. (B) The original (reweighted) and modified (no reweighting) PMF profiles of the distance between the $\mathrm{N}$ atom of benzamidine ligand and $\mathrm{CG}$ atom of protein residue Asp189. (C) Reweighted and (D) modified 2D PMF profiles of the benzamidine:N - Asp189:CG and Trp215:NE - Asp189:CG atom distances. The PMF profiles were calculated by combining all five 1000 ns LiGaMD_Dual simulations. 
Fig. 6 Six representative low-energy conformational states as identified from PMF profiles of benzamidine (BEN) ligand (spheres) binding to trypsin (blue ribbons): (A) "Bound (B)", (B) “Intermediate 1 (I1)", (C) "Intermediate 2 (I2)", (D) "Intermediate 3 (I3)", (E) "Unbound (U1)", and (F) "Unbound (U2)". Reference X-ray conformations of the ligand and protein (PDB: 3PTB) are shown in green spheres and grey ribbons, respectively. Protein residues Asp189 and Trp215 that are important for ligand binding and those in the catalytic triad (His57, Asp102 and Ser214) are highlighted in thick sticks.

Fig. 7 Pathways of the benzamidine (BEN) ligand (sticks) in trypsin (ribbons) obtained from structural clustering of five 1000 ns LiGaMD_Dual simulations: (A) Sim1, (B) Sim2, (C) Sim3, (D) Sim4 and (E) Sim5. The ligand is represented by sticks that are colored by reweighted PMF values of the ligand clusters in a blue $(0 \mathrm{kcal} / \mathrm{mol})$-white $(7.5 \mathrm{kcal} / \mathrm{mol})$-red $(15.0 \mathrm{kcal} / \mathrm{mol})$ scale. X-ray conformation of the ligand is shown in green spheres for reference. Protein residues Asp189 and Trp215 that are important for ligand binding and those in the catalytic triad (His57, Asp102 and Ser214) are highlighted in thick sticks. Other negatively charged residues in the protein including Glu70, Asp71, Glu77, Glu80 and Asp153 are shown in thin sticks. The protein loops containing residues Asp189 and Trp215 are highlighted in yellow and green, respectively. 
Fig. 1

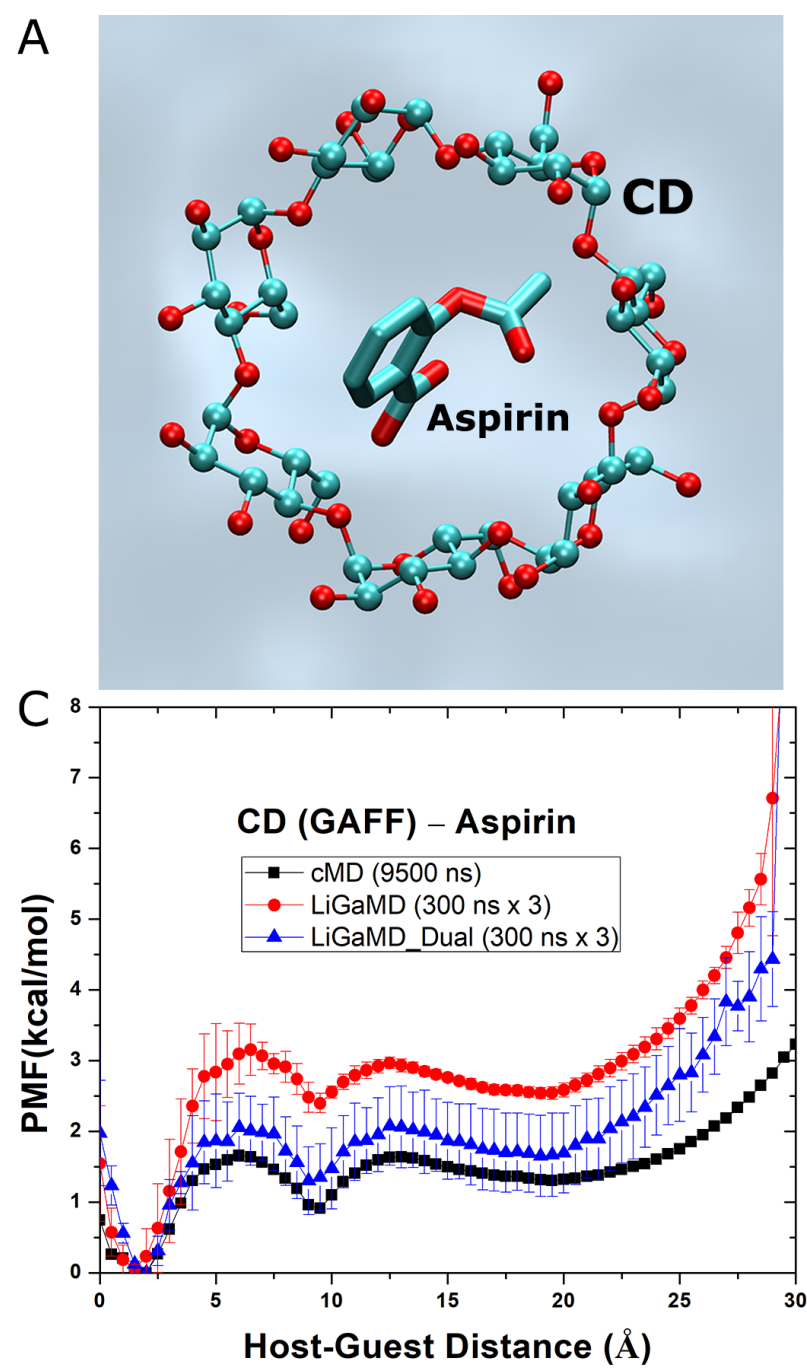

B
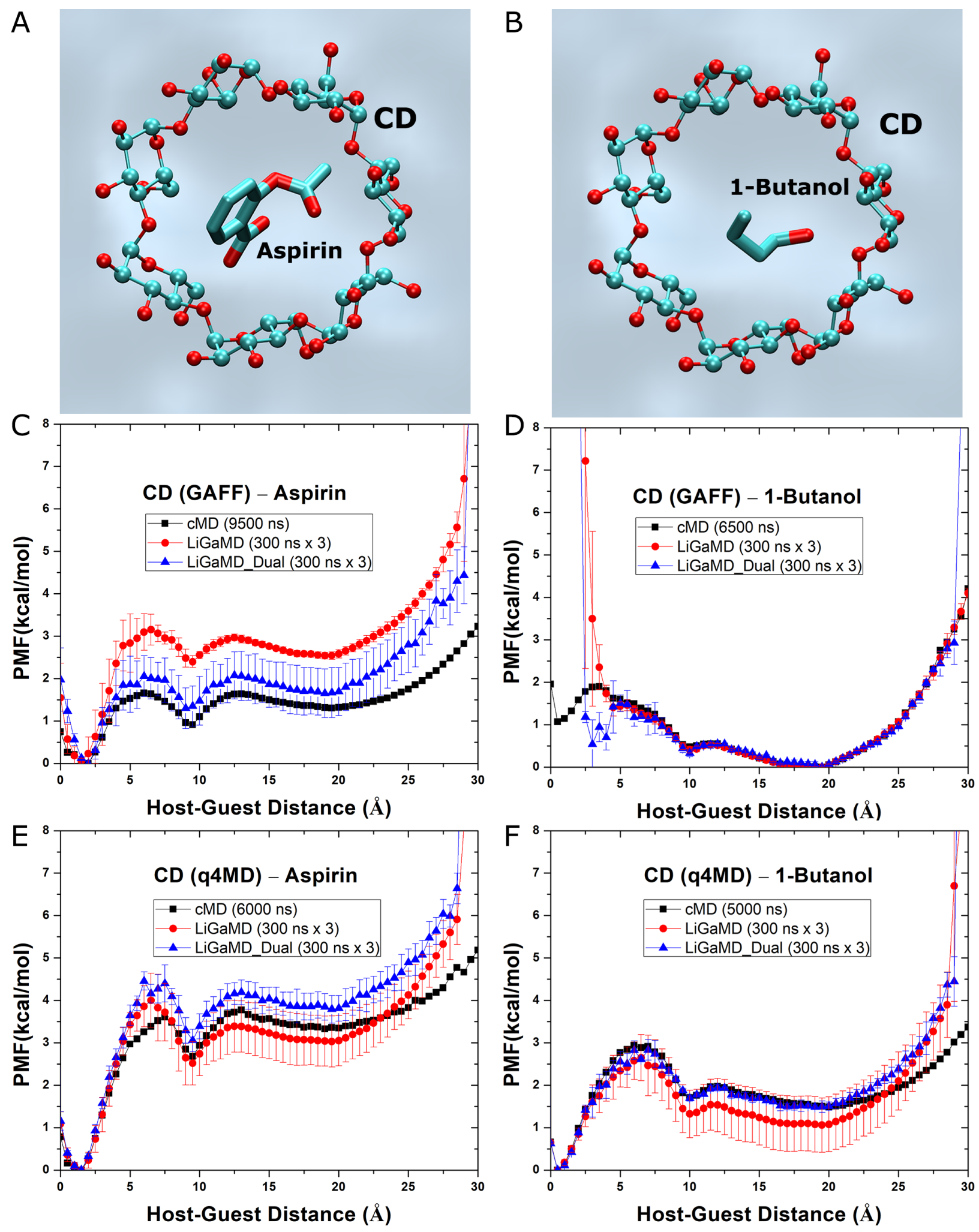
Fig. 2
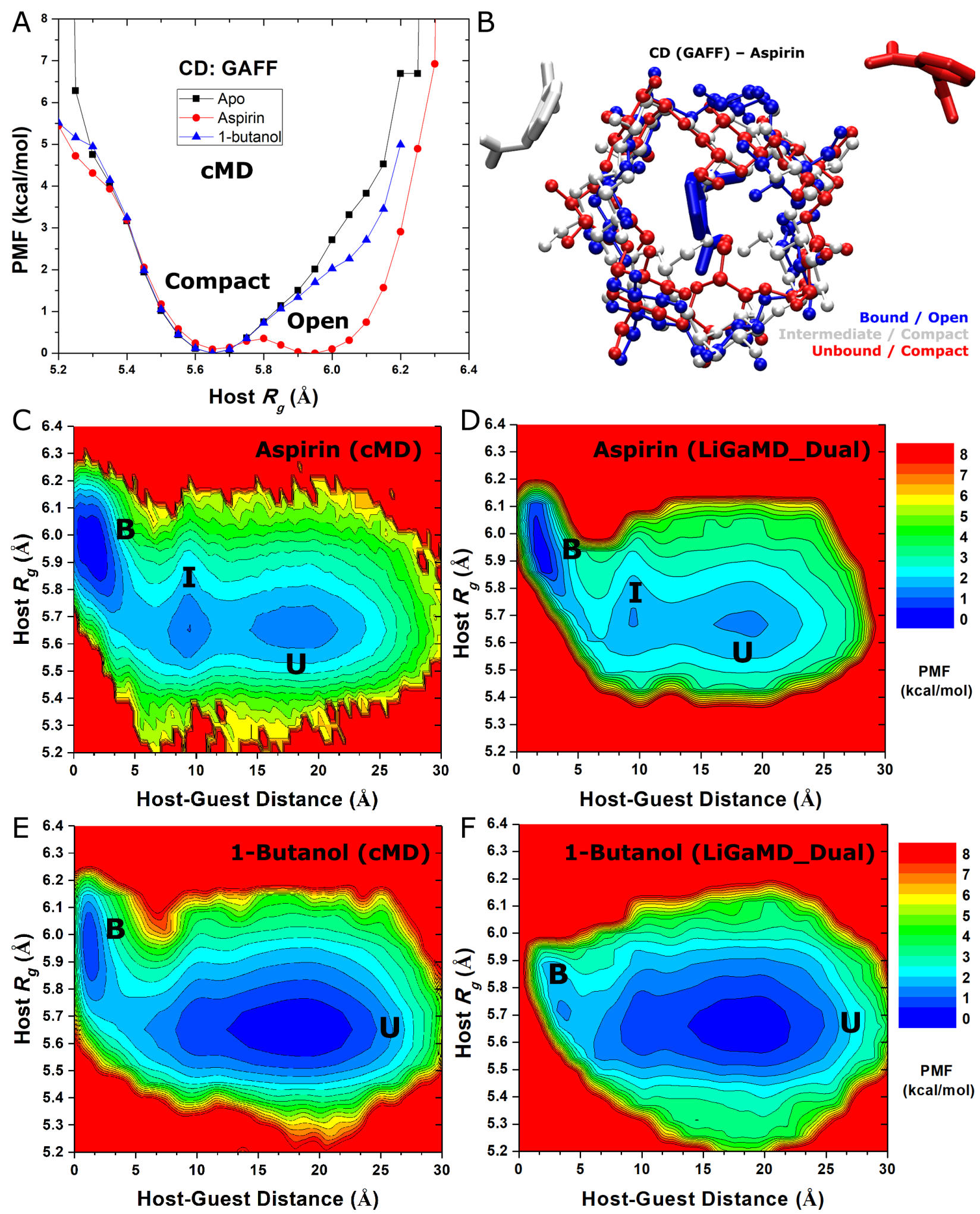
Fig. 3
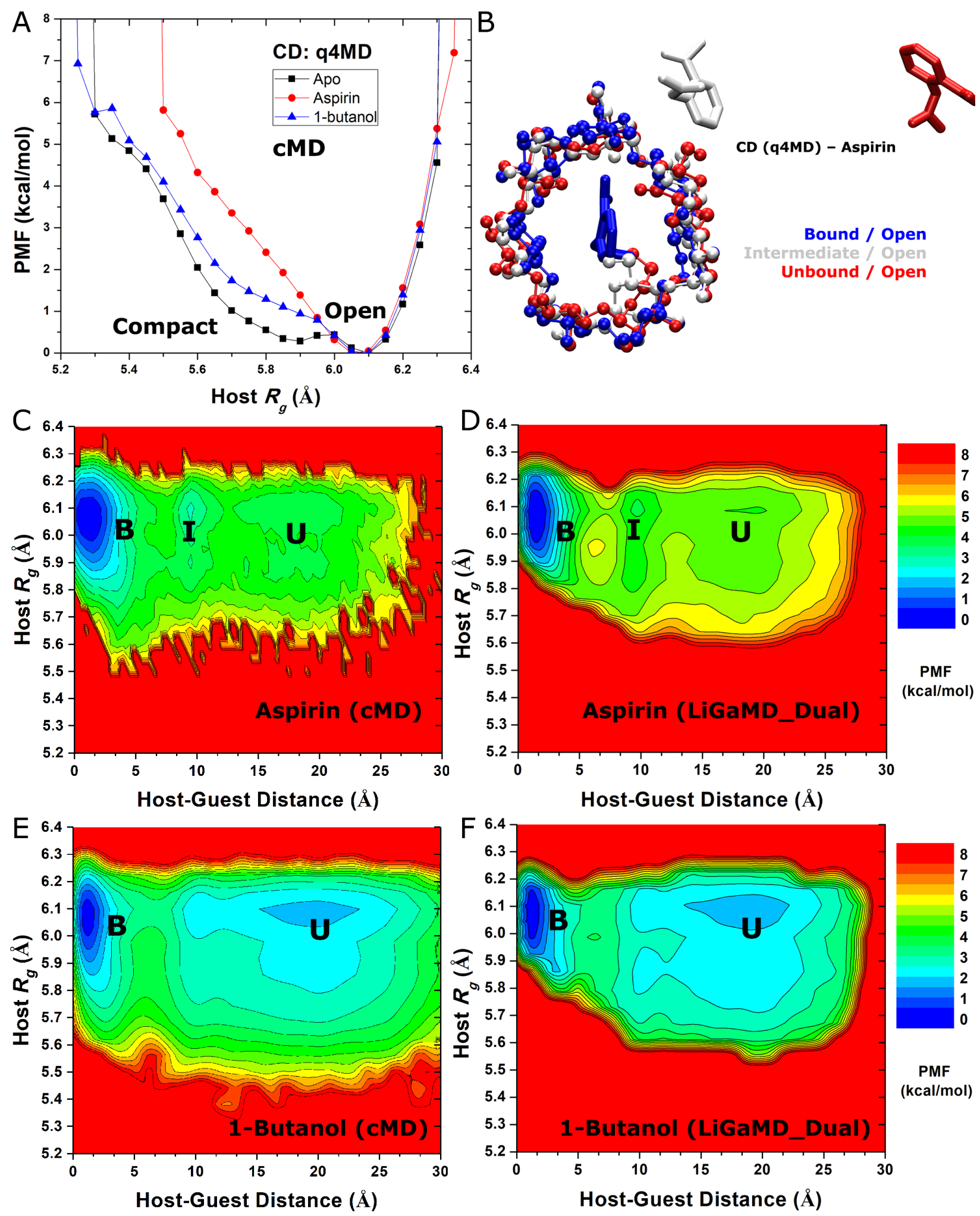
Fig. 4
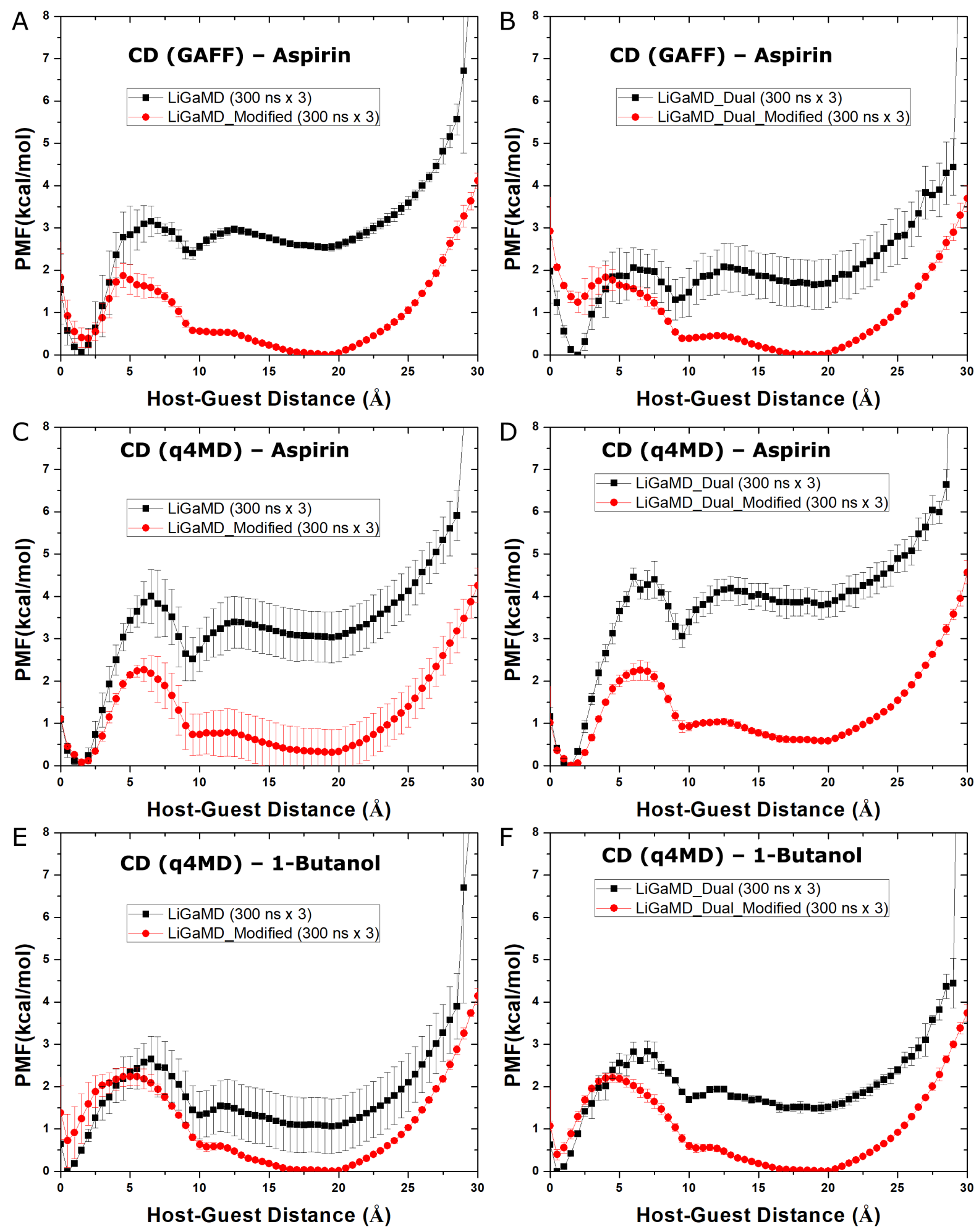
Fig. 5
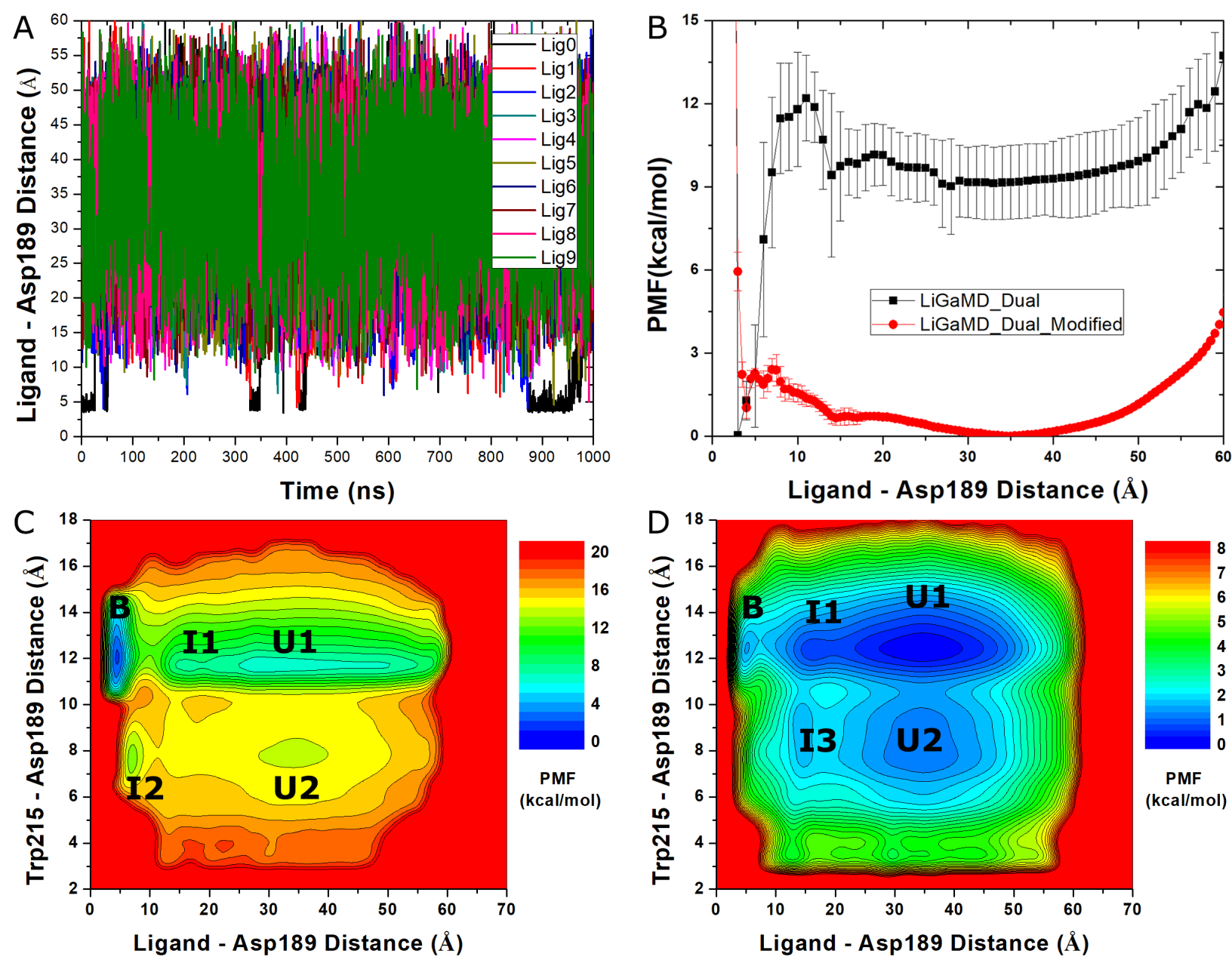
Fig. 6
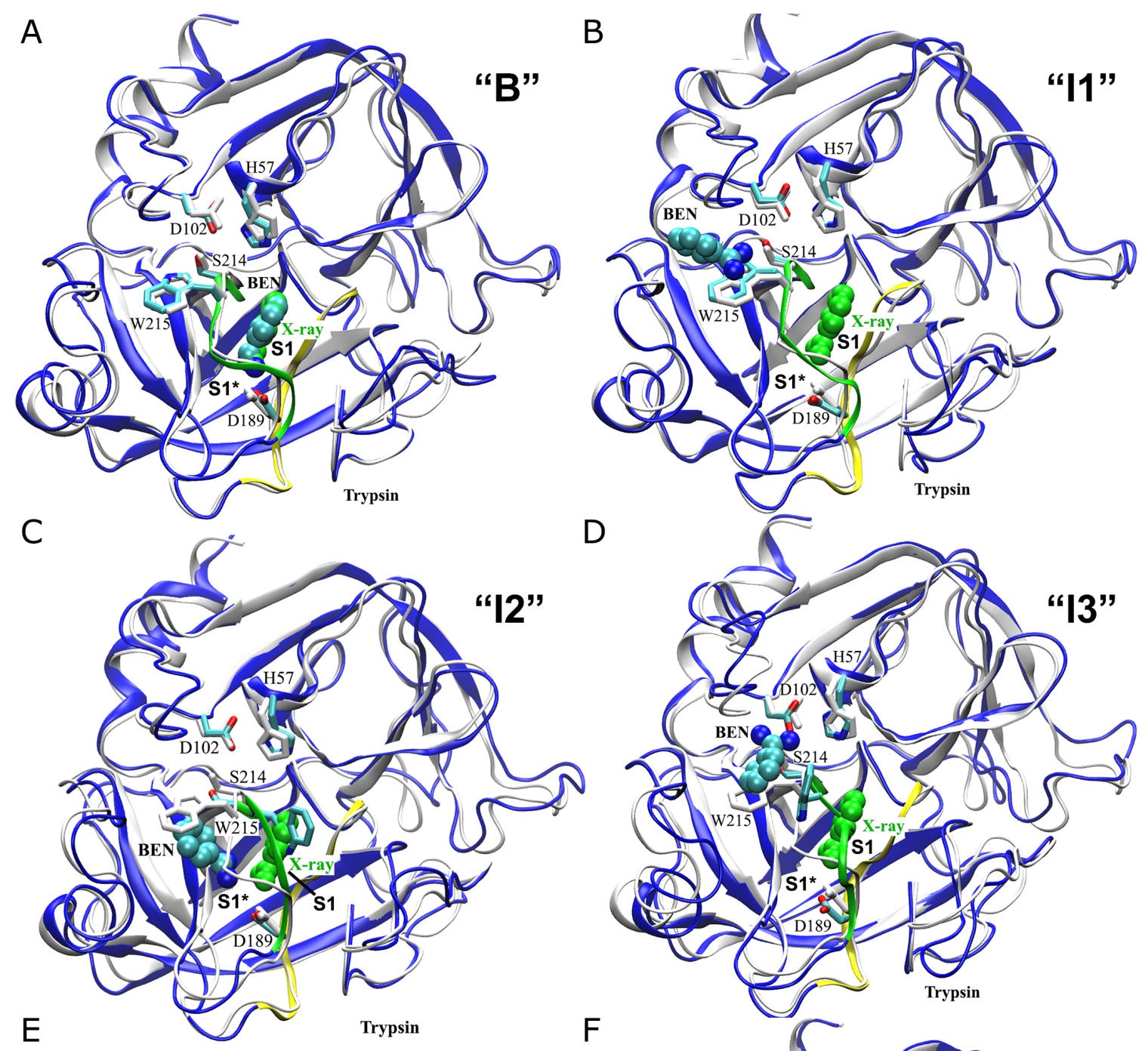

D
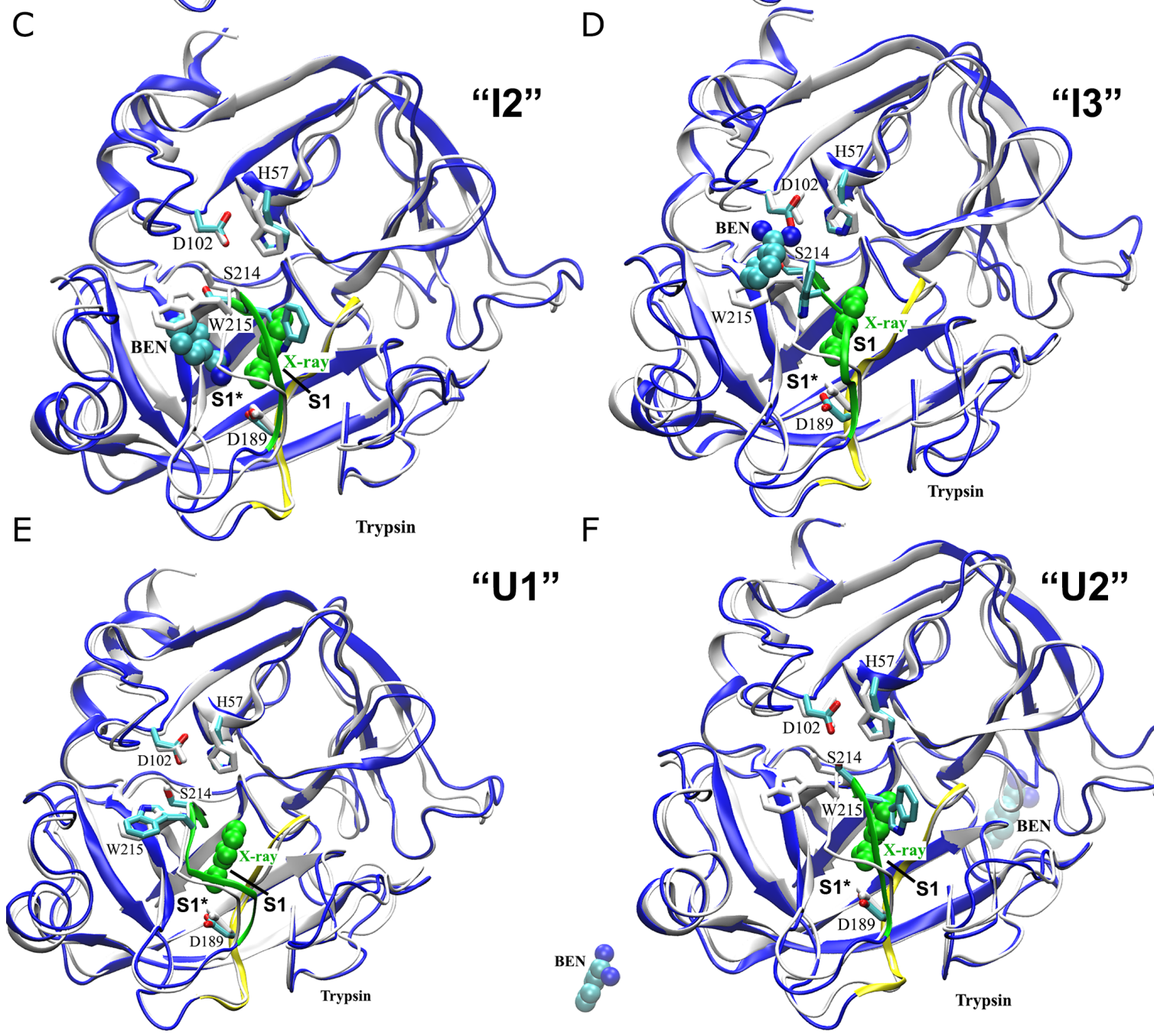

"U1"

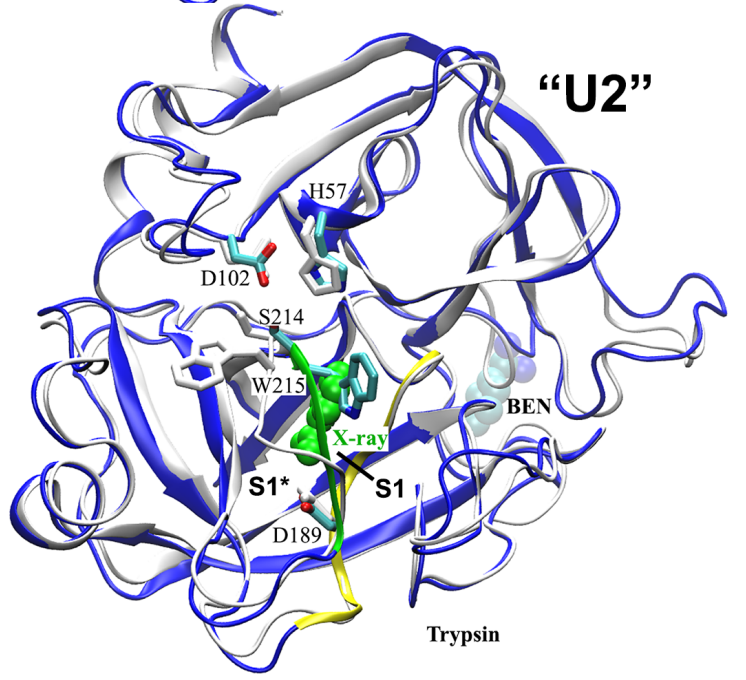


bioRxiv preprint doi: https://doi.org/10.1101/2020.04.20.051979; this version posted April 21, 2020. The copyright holder for this preprint (which was not certified by peer review) is the author/funder, who has granted bioRxiv a license to display the preprint in perpetuity. It is made available under aCC-BY-NC-ND 4.0 International license.

\section{Fig. 7}

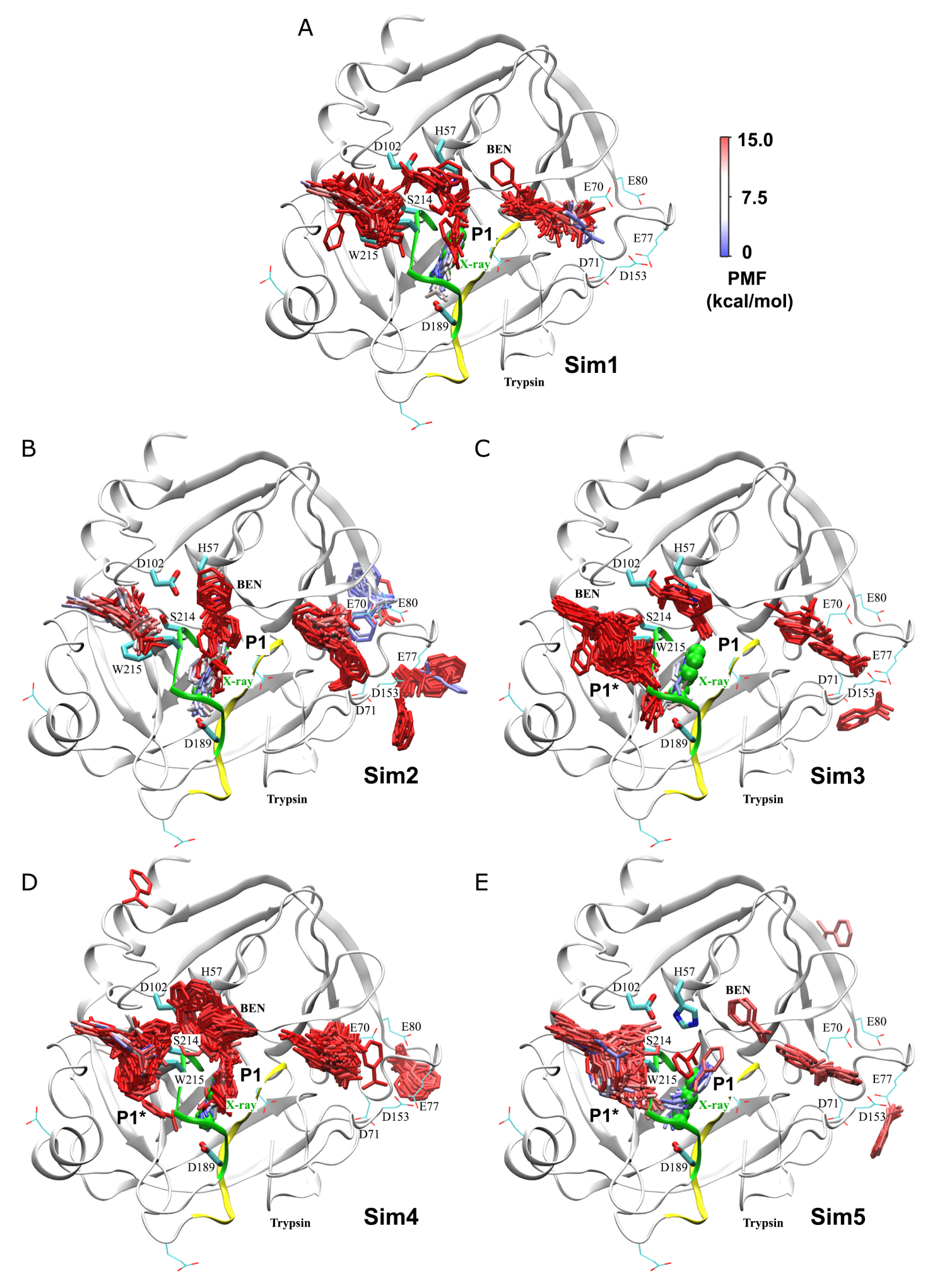




\section{TOC graphic}
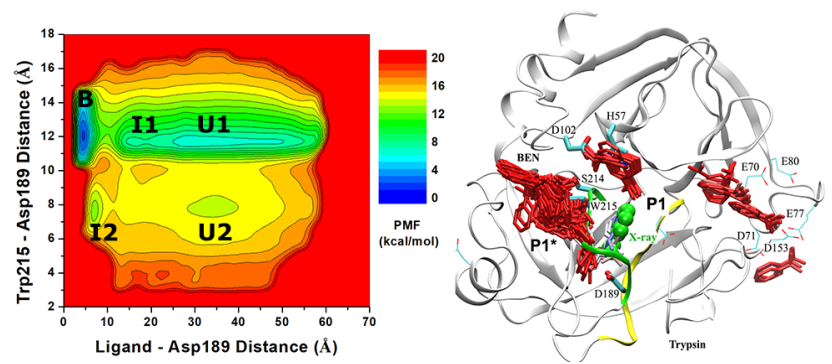

All-atom ligand Gaussian accelerated molecular dynamics (LiGaMD) simulations captured repeptitive binding of the benzamidine (BEN) ligand to the trypsin model protein, which enabled us to characterize the ligand binding free energy profiles, pathways and kinetic rate constants. 